ARTICLE

Received 28 Nov 2015 | Accepted 16 Feb 2016 | Published 30 Mar 2016

DOI: $10.1038 /$ ncomms11056

OPEN

\title{
Loss of MAX results in meiotic entry in mouse embryonic and germline stem cells
}

\author{
Ayumu Suzuki ${ }^{1}$, Masataka Hirasaki ${ }^{1}$, Tomoaki Hishida1,2, Jun Wu², Daiji Okamuraa,3, Atsushi Ueda ${ }^{1}$, \\ Masazumi Nishimoto ${ }^{1}$, Yutaka Nakachi ${ }^{4,5}$, Yosuke Mizuno ${ }^{5}$, Yasushi Okazaki ${ }^{4,5}$, Yasuhisa Matsui6,7, \\ Juan Carlos Izpisua Belmonte ${ }^{2} \&$ Akihiko Okuda $^{1}$
}

Meiosis is a unique process that allows the generation of reproductive cells. It remains largely unknown how meiosis is initiated in germ cells and why non-germline cells do not undergo meiosis. We previously demonstrated that knockdown of Max expression, a gene encoding a partner of MYC family proteins, strongly activates expression of germ cell-related genes in ESCs. Here we find that complete ablation of Max expression in ESCs results in profound cytological changes reminiscent of cells undergoing meiotic cell division. Furthermore, our analyses uncovers that Max expression is transiently attenuated in germ cells undergoing meiosis in vivo and its forced reduction induces meiosis-like cytological changes in cultured germline stem cells. Mechanistically, Max depletion alterations are, in part, due to impairment of the function of an atypical PRC1 complex (PRC1.6), in which MAX is one of the components. Our data highlight $M A X$ as a new regulator of meiotic onset.

\footnotetext{
${ }^{1}$ Division of Developmental Biology, Research Center for Genomic Medicine, Saitama Medical University, Yamane Hidaka, Saitama 350-1241, Japan. ${ }^{2}$ Gene Expression Laboratory, Salk Institute for Biological Studies, 10010 North Torrey Pines Road, La Jolla, California 92037, USA. ${ }^{3}$ Universidad Católica San Antonio de Murcia (UCAM) Campus de los Jerónimos, No. 135, Guadalupe, 30107 Murcia, Spain. ${ }^{4}$ Division of Translational Research, Research Center for Genomic Medicine, Saitama Medical University, Yamane Hidaka, Saitama 350-1241, Japan. ${ }^{5}$ Division of Functional Genomics and Systems Medicine, Research Center for Genomic Medicine, Saitama Medical University, Yamane Hidaka, Saitama 350-1241, Japan. ${ }^{6}$ Cell Resource Center for Biomedical Research, Institute of Development, Aging and Cancer, Tohoku University, Sendai 980-8575, Japan. ${ }^{7}$ Japan Agency for Medical Research and Development and Development-Core Research for Evolutionary Science and Technology (AMED-CREST), Tokyo 100-0004, Japan. Correspondence and requests for materials should be addressed to J.C.I.B. (email: belmonte@salk.edu) or to A.O. (email: akiokuda@saitama-med.ac.jp).
} 
$\mathrm{M}$ eiosis is a specialized cell division process that produces gametes with a haploid genome ${ }^{1}$. Up to date, several reports have demonstrated significant conservation among species in the molecular basis underlying meiosis ${ }^{2}$. However, the molecular mechanisms that allow switching from mitotic to meiotic cell division in germ cells do not appear to be conserved across species or even between sexes within the same species. Stra8, whose expression is upregulated by retinoic acid (RA), has been shown to play crucial roles in premeiotic DNA replication ${ }^{3-6}$ and constitutes a physiological and crucial regulator of the initiation of meiosis in both sexes ${ }^{7-10}$. In addition to the RA-Stra8 signalling pathway, it is plausible that unknown negative regulator of meiosis may prevent its premature entry in germ cells or ectopic induction in non-germ cells.

Recently, we demonstrated that knockdown of expression of the Max gene, encoding an indispensable partner for transcription factor c-MYC in embryonic stem cells (ESCs), leads to strong induction of germ cell-related gene expressions ${ }^{11}$. Notably, loss of Max expression in ESCs does not significantly alter the expression levels of primordial germ cell (PGC) specification genes such as Blimp1 and Stella (also known as $P r d m 1$ and $D p p a 3$, respectively), but rather selectively upregulates expression of genes related to meiotic cell division ${ }^{11}$. This preferential expression of meiosis-related genes in Max knockdown ESCs prompted us to explore the possibility that MAX might be part of the mechanism that safeguards meiosis by controlling the physiological timing of meiosis onset and preventing ectopic meiosis.

In this study, we found that Max depletion in ESCs not only upregulated the expression of meiosis-related genes but also induced the cytological changes reminiscent of germ cells at leptotene and zygotene stages of meiosis. Furthermore, our data revealed that these cytological changes even occurred in ESCs cultured in stringent $2 \mathrm{i}$ condition that renders ESCs refractory to cellular differentiation. This implies a direct conversion of Max-null ESCs to a meiosis-like state bypassing PGC differentiation. Moreover, we demonstrated that Stra8, but not Blimp1, was required for these cytological changes. Our analyses revealed that the Max gene undergoes a strong decline in expression during physiological meiosis in both male and female germs cells. Forced reduction of Max expression levels in germline stem cells (GSCs) ${ }^{12,13}$ by lentivirus-mediated knockdown induced meiosis-like cytological changes. Our findings in Max-null ESCs may reflect a physiological role for MAX during in vivo meiosis. Max-null ESCs may serve as a useful in vitro tool for studying the molecular mechanisms governing mitotic versus meiotic cell divisions. Mechanistically, our data indicate that these cytological changes are the result of loss of function of a variant PRC1 complex (PRC1.6) in which MAX is a component ${ }^{14,15}$.

\section{Results}

Induction of meiosis-related genes in Max-null ESCs. Our previous RNA interference screen uncovered Max as a strong suppressor of the expression of germ cell-related genes such as $D d x 4$ (also known as $M v h$, mouse Vasa homologue) and Dazl, in ESCs $^{11}$. Owing to residual Max expression in knockdown experiments, we hypothesized that Max knockout may elicit more profound effects. We first analysed our Max-null ESC transcriptome data set (GSE27881) reported previously. In these cells, the endogenous Max gene was homozygously disrupted, and doxycycline (Dox)-regulatable expression of MAX was introduced using the tetracycline-off system ${ }^{16}$. Consistent with our hypothesis, expression levels of many germ cell-related genes were elevated in Max-null ESCs, of which most are meiosis-related (Fig. 1a,b and Supplementary Fig. 1). Some of the meiotic genes, such as Stra 8 and Dazl, showed progressive increase in the expression levels following Dox treatment, while expression of others, such as Hormad1 and Stk31, peaked at around day 4 and abruptly dropped thereafter. However, it is unknown whether the latter expression dynamics are caused by a negative feedback loop of regulation of these meiotic genes or simply a consequence of extensive cell death phenotype associated with meiotic-like Max-null ESCs. Unlike meiosis-related genes, PGC specification genes such as Blimp1 and Dppa3 did not show appreciable alterations in their expression levels on Dox treatment. We also noted that the expression levels of genes encoding meiosis-specific cohesion components, such as Rec8 and $S m c 1 b$, were elevated, to a lesser extent, in Dox-treated cells compared with untreated controls. We also examined the expression of genes encoding key regulators of DNA recombination and found most of them remained unchanged, although a modest increase was evident in Spo11 expression. These results implied that Max-null ESCs exhibited a partial, but not complete, gene expression pattern characteristic of meiotic cells (Supplementary Fig. 1). In addition, we also observed increased expression of two-cell embryo signature genes such as Zscan4 and Dub1 (refs 17,18; Fig. 1a,b and Supplementary Fig. 1). However, flow cytometric analyses of Dox-inducible Max-null ESCs bearing a DsRed reporter gene, which faithfully recapitulates the endogenous Zscan4 expression profile with an aid of $5^{\prime}$-flanking region of the gene, indicated that DsRed- and STRA8-positive cells, both of which became prominent after Max depletion in ESCs, did not significantly overlap, but were rather mutually exclusive (Supplementary Fig. 2). These data implied that meiosis-like induction and activation of two-cell embryo gene signatures are independent phenomena. As expected, gene ontology (GO) analysis revealed over-representation of genes related to meiosis and sexual reproduction (Fig. 1c).

Max-null ESCs show meiosis-like cytological changes. Expression of meiosis-related genes led us to examine cytological changes in Max-null ESCs. To this end we performed immunocytochemical analyses with an antibody against SYCP3, one of the major components of the synaptonemal complex ${ }^{19,20}$. After 8 days of Dox treatment, about $4-5 \%$ of Max-null ESCs showed a SYCP3-immunostaining pattern that is reminiscent of germ cells undergoing meiosis (Fig. 2a,b). We observed about 20\% STRA8-positive cells among all Max-null ESCs treated with Dox for 6 days (Supplementary Fig. 2). Most STRA8-positive cells, however, failed to proceed to the stage acquiring a meiosis-like SYCP3-staining pattern. To assess the stages of meiosis in meiosis-like cells from Max-null ESCs, we established a criterion to distinguish the stages based on the average length of SYCP3-stained regions observed in the chromosomes of germ cells in seminiferous tubules of the testis (Supplementary Fig. 3). Our analyses indicated that meiosis-like cells derived from Max-null ESCs following 6 days' Dox treatment were mostly preleptotene-like, with some cells progressed to the leptotene-like state. And longer Dox treatment (8 or 10 days) increased the percentages of these cells and also yielded cells bearing SYCP3-staining patterns similar to germ cells at zygotene stages of meiotic cell division (Fig. 2b). However, no cell was scored as pachytene-like even with extended Dox treatment (Fig. 2a,b). To confirm these observations, we conducted co-staining with antibodies against SYCP3 together with other meiotic markers. First, similar to the normal progression of meiosis, we found that STRA8 expression was more enriched in cells representing the early stages of meiosis and much less in cells at a more advanced 


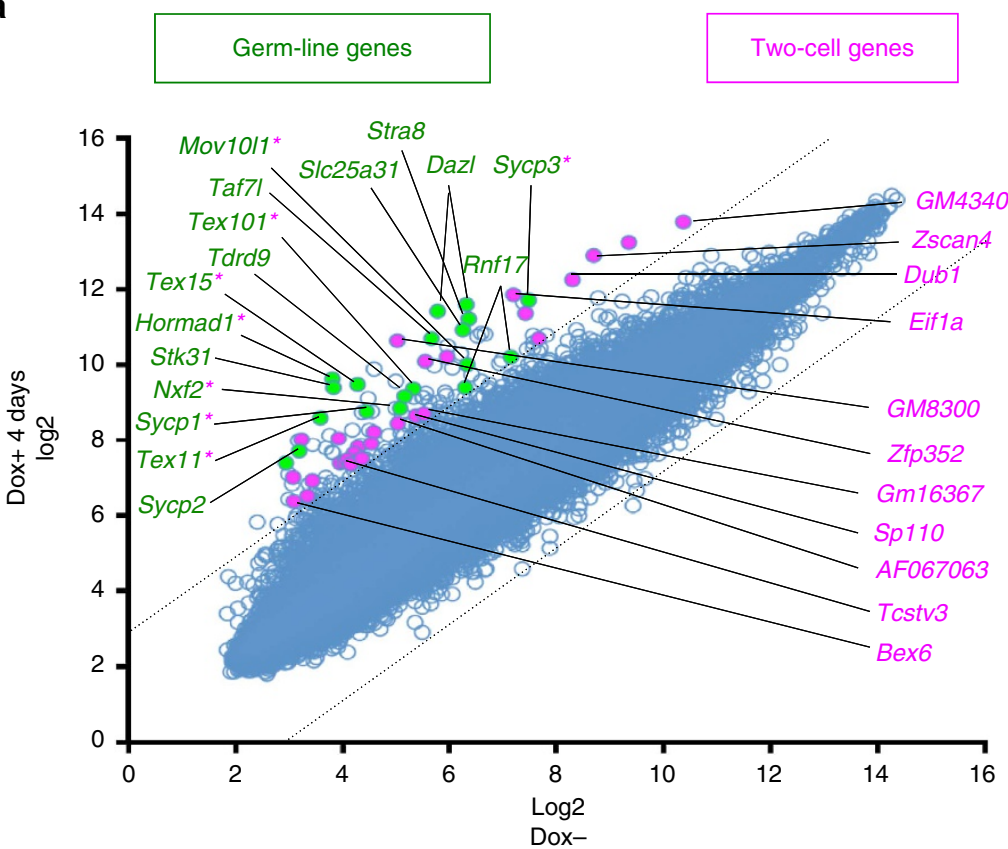

b

Most up-regulated 10 gene-IDs at 4 days after dox addition

\begin{tabular}{|c|c|c|c|c|c|c|c|c|}
\hline \multirow[b]{2}{*}{ Probe ID } & \multirow[b]{2}{*}{ Lanking } & \multirow[b]{2}{*}{ Gene symbol } & \multicolumn{6}{|c|}{ Days after dox addition } \\
\hline & & & $1 \mathrm{~d}$ & $2 \mathrm{~d}$ & $3 d$ & $4 \mathrm{~d}$ & $6 \mathrm{~d}$ & $8 \mathrm{~d}$ \\
\hline 1450292_a_at & 1 & Hormad1* & 13.81 & 3.21 & 31.74 & 56.20 & 12.77 & 8.16 \\
\hline 1419542_at & 2 & Dazl & 5.00 & 1.71 & 21.09 & 49.16 & 43.23 & 41.21 \\
\hline 1444529_at & 3 & Gm8300 & 2.24 & 2.54 & 25.96 & 48.79 & 41.80 & 36.99 \\
\hline 1454138_a_at & 4 & Stk31 & 12.49 & 3.08 & 24.16 & 47.42 & 16.22 & 8.69 \\
\hline 1419665_a_at & 5 & Nupr1 & 6.71 & 24.85 & 52.85 & 39.55 & 1.52 & 0.72 \\
\hline 1449502_at & 6 & Dazl & 4.11 & 1.27 & 15.49 & 38.68 & 47.85 & 42.84 \\
\hline 1420719_at & 7 & Tex15* & 3.91 & 3.81 & 12.08 & 37.09 & 33.16 & 21.74 \\
\hline 1420433_at & 8 & Taf7l & 4.71 & 1.13 & 21.81 & 32.48 & 42.20 & 37.63 \\
\hline 1419729_at & 9 & Tex11* & 6.68 & 3.86 & 25.06 & 32.02 & 9.35 & 6.38 \\
\hline 1420568_at & 10 & Stra8 & 2.96 & 1.67 & 7.72 & 29.15 & 38.90 & 45.42 \\
\hline
\end{tabular}

C

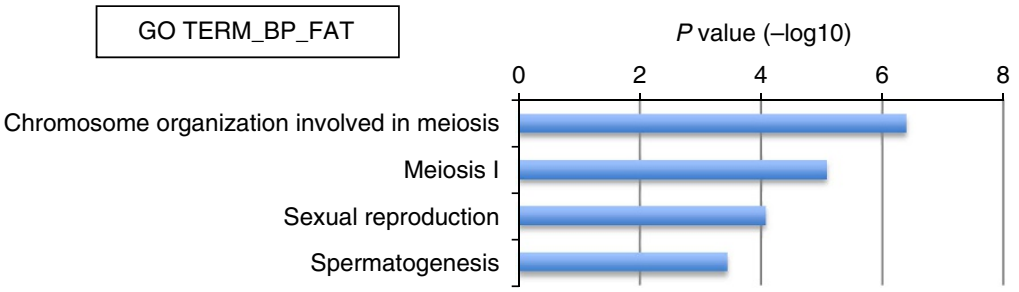

Figure 1 | Elevation of germ cell- and two-cell embryo-related genes in ESCs subjected to Max expression ablation. (a) Scatter plots of DNA microarray data from untreated and Dox-treated Max-null ESCs for 4 days using our previously deposited DNA microarray data under accession number GSE27881. Among genes with highly elevated expression in Dox-treated Max-null ESCs compared with that in untreated control cells (eightfold or higher), genes related to germ cells (green) and/or two-cell embryos denoted by Macfarlan et al. ${ }^{18}$ (pink) are highlighted. Genes belonging to both categories are green with pink asterisks added to the gene names. (b) List of probe identifications (IDs) that showed the most conspicuous upregulation (top 10) in Max-null ESCs treated with Dox for 4 days (4d) and their expression changes during Max expression ablation in ESCs. Dazl is duplicated with different probe IDs. (c) GO analyses of genes showing more than eightfold higher expression in Dox-treated Max-null ESCs using DAVID software. The $P$ value cutoff was set to $<1 \times 10^{-3}$.

stage with a zygotene-like SYCP3-staining pattern (Fig. 2c). We also found that cells with a leptotene-like SYCP3-staining pattern showed a strong $\gamma \mathrm{H} 2 \mathrm{AX}$ signal, similar to meiotic cells at this stage (Fig. 2d). Furthermore, REC8 and SYCP3 were co-localized in some, but not all, of the cells with a zygotenelike SYCP3-staining pattern (Supplementary Fig. 4a), likely due to less conspicuous induction of $\operatorname{Rec} 8$ compared with that of other meiotic marker genes such as Stra 8 and Sycp3 (Supplementary Fig. 1). We also conducted immunostaining with an antibody against SYCP1 that is known to play a crucial role in the formation of the synaptonemal complex at the pachytene stage of meiosis, in which two paired homologous chromosomes (four chromosomes in total) are brought into a juxtaposition as a prerequisite step for the subsequent crossover $^{21-23}$. Although SYCP1 expression was detected in some cells, we did not observe extensive overlap of SYCP1 signals with that of the SYCP3 on chromosomes (Supplementary Fig. 4b). These results were consistent with the data shown in Fig. 2b and imply that Max deficiency arrests ESCs at the crux of the pachytene stage of meiosis-like processes. Because aurora kinase is a known critical regulator of meiosis ${ }^{24}$, we examined changes in the expression of aurora kinase A, B and C in Max-null ESCs by western blot 
a

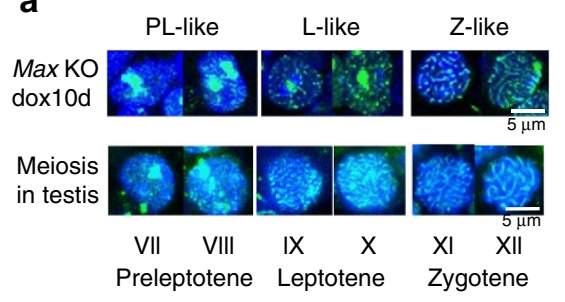

b

C

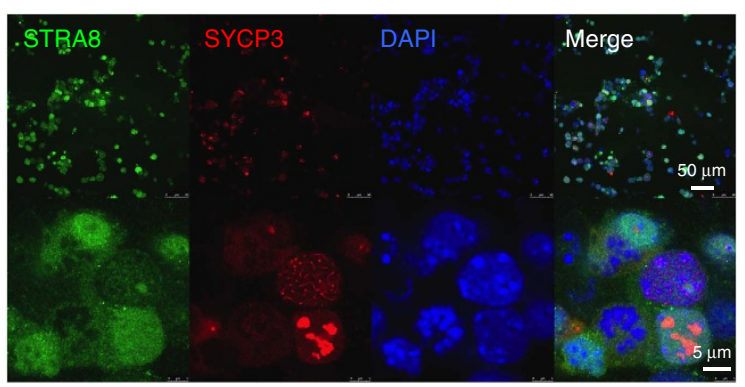

d

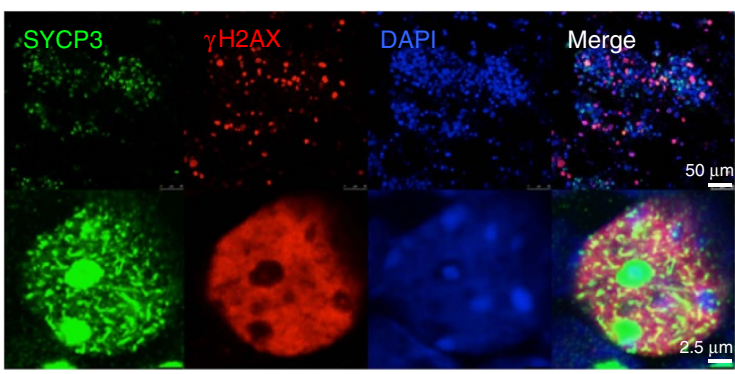

Figure 2 | Cytological changes reminiscent of cells undergoing meiotic cell division processes. (a) Meiosis-like SYCP3-staining patterns in Maxnull ESCs treated with Dox for 10 days. The meiosis-like SYCP3-staining patterns in Max-null ESCs were classified as preleptotene (PL)-, leptotene (L)- and zygotene (Z)-like patterns according to the criteria shown in Supplementary Fig. 3. (b) Frequency of preleptotene-, leptotene- and zygotene-like SYCP3-staining patterns in Max-null ESCs at the indicated days after Dox administration. (c) Co-immunostaining analyses of Max-null ESCs treated with Dox for 9 days with antibodies against SYCP3 and STRA8. (d) Max-null ESCs treated with Dox for 10 days were co-immunostained for SYCP3 and $\gamma \mathrm{H} 2 \mathrm{~A} . \mathrm{X}$.

analyses. All three aurora kinases exhibited reductions in their protein expression levels, although the reduction of aurora kinase C was marginal (Supplementary Fig. 5a). Next, we examined the effect of a pan-aurora kinase inhibitor, VX680, and found that it did not noticeably alter the magnitude of meiosis-like changes in Max-null ESCs (Supplementary Fig. 5b,c). Because aurora kinase $\mathrm{C}$ is the only aurora kinase with an unequivocal contribution to meiosis among the three aurora kinases and localization of aurora kinase $\mathrm{C}$ at chromocentres becomes apparent at the diplotene stage ${ }^{24}$, no apparent effect of the aurora kinase inhibitor may be reasonable in our system in which the meiotic process was arrested at or before the pachytene stage (Fig. 2). Next, we examined whether loss of Max expression in ESCs was accompanied by alterations in genomic DNA methylation levels. To this end we compared the levels of 5 -methyl cytosine $(5 \mathrm{mC})$ and 5-hydroxymetyl cytosine $(5 \mathrm{hmC})$ between Dox-untreated and -treated ESCs for 8 days. Although $5 \mathrm{mC}$ levels remained rather constant on Max depletion, our analyses demonstrated an elevation in the levels of $5 \mathrm{hmC}$ by about twofold in Dox-treated Max-null ESCs (Supplementary Fig. 6a). We also examined the methylation status of imprinting genes because erasure of imprinting is one of the hallmarks of germ cell development. We found that differentially methylated regions (DMRs) of maternally methylated Snrpn and $I g f 2 r$ genes were almost completely demethylated in Max expression-ablated ESCs, implying that activation of the meiotic programme in these cells was coupled with imprinting erasure (Supplementary Fig. 6b,c). We also found similar, but less significant, induction of demethylation in one of the DMRs in the paternally methylated H19 gene (Supplementary Fig. 6d). The almost complete methylation of DMRs in the H19 gene of Dox-untreated ESCs reflects the usual DNA methylation status of this locus in mouse ESCs cultured in conventional mouse ESC medium ${ }^{25-27}$.

Vitamin C enhances meiosis-related changes in Max-null ESCs. Since apoptosis is a prominent phenotype of Max-null ESCs, we investigated whether apoptosis might be triggered by meiosis-like changes or elevated levels of reactive oxygen species in Max-null ESCs ${ }^{16}$. Towards this end we treated Max-null ESCs with vitamin $\mathrm{C}$, a compound that has been described both as an antioxidant and as a potentiating factor in meiosis through activation of ten-eleven translocation 1 (Tet 1$)^{28,29}$. Vitamin C treatment of Max-null ESCs did not reduce but instead significantly accelerated cell apoptosis (Fig. 3a). This was accompanied by elevated Stra8 expression (Fig. 3b). Vitamin C treatment also increased the frequency of meiosis-like SYCP3staining patterns and yielded cells representing more advanced stages of meiosis compared with Dox treatment alone (Fig. 3c,d). These results implied that the meiosis-related changes rather than reactive oxygen species in Max-null ESCs are closely linked to apoptosis. To further explore the relationship between the meiosislike changes and apoptotic cell death in Max-null ESCs, we suppressed the levels of apoptosis by treatment with the caspase inhibitor Z-VAD-FMK. We found that Z-VAD-FMK treatment increased the frequency of cells with meiosis-like changes, especially cells with preleptotene- and leptotene-like SYCP3staining patterns (Supplementary Fig. 7). These observations implied that a substantial portion of meiotic-like Max-null ESCs were eliminated at rather early stages before proceeding to more advanced stages of meiosis. Apoptosis occurred likely as a result of the ESC culture condition, which is different from the niche environment found within the seminiferous tubules of the testis and the female genital ridge.

Meiosis-like changes in Max-null ESCs depend on Stra8 gene. To explore the relationship between the induction of Stra8 gene expression and the meiosis-like SYCP3-staining patterns in our system, we knocked out the Stra8 gene in Max-null ESCs using the CRISPR-Cas9 system $^{30}$ (Fig. 4a). Immunocytochemical (Fig. 4b) and western blot (Fig. 4c) analyses confirmed that Max depletion-mediated induction of Stras expression did not occur in Stra8-knockout ESCs. More importantly, loss of Stra8 gene functions resulted in marked suppression of incidences of meiosis-like SYCP3-staining patterns in Dox-treated Max-null ESCs (Fig. 4d). We also found that disruption of the Stra8 gene led to a significant reduction in $\gamma \mathrm{H} 2 \mathrm{AX}$ signals, a characteristic feature observed strongly at leptotene stages of meiosis (Fig. 4e). These data implied that, similar to normal meiotic cell division, expression of Stra8 is indispensable for induction of meiotic-like patterns in Max-null ESCs ${ }^{3-6}$. It is also worth noting that Stra8 knockout not only impaired the cytological changes reminiscent of meiosis but also significantly alleviated apoptotic cell death phenotype associated with Max knockout (Fig. 4f,g). Therefore, these results further suggest that the extensive cell death observed in Max-null ESCs is, at least in part, due to induction of meiosis. 

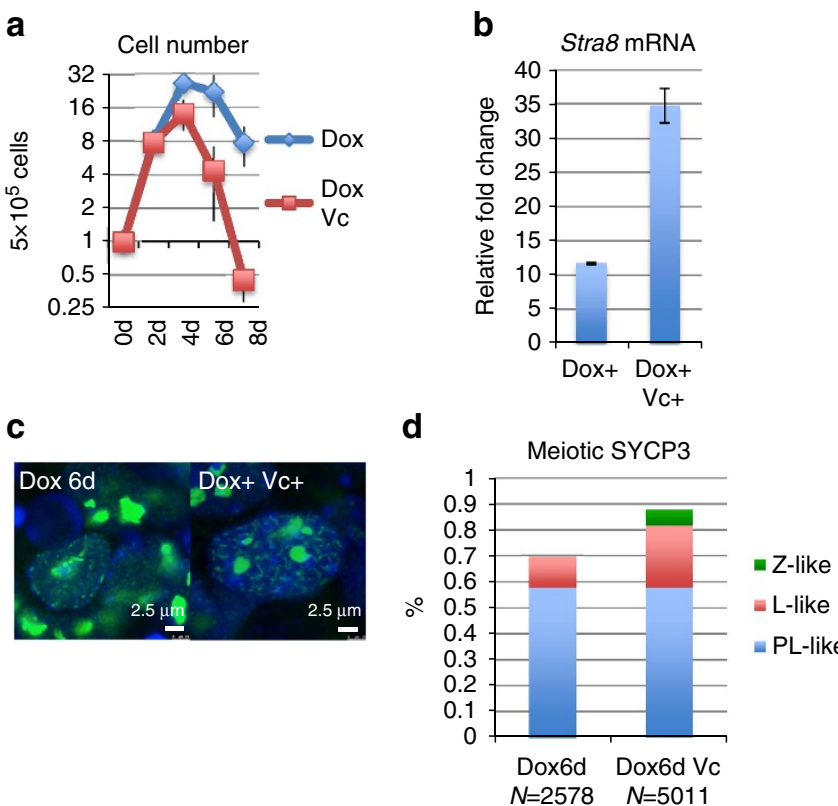

Figure 3 | Vitamin C enhances meiosis-related changes in Max expression-ablated ESCs. (a) Total number of viable Dox-treated Max-null ESCs cultured in the presence or absence of vitamin $C$ at the indicated days after Dox addition. The number of cells at day 0 (Od) was arbitrarily set to one $(n=3$, means \pm s.d.). (b) Expression levels of the Stra8 gene in Doxtreated Max-null ESCs cultured in the presence or absence of vitamin C for 4 days (4d). The expression level of the Stra8 gene in untreated control Max-null ESCs was arbitrarily set to one $(n=3$, means \pm s.d.).

(c) Representative meiosis-like SYCP3-staining patterns in Dox-treated Max-null ESCs cultured in the absence or presence of vitamin $C$ for 6 days (6d). (d) Frequency of meiosis-like SYCP3-staining patterns in Max-null ESCs treated only with Dox for 6 days or together with vitamin C.

Meiosis-like changes in Max-null ESCs under $2 \mathrm{i}$ condition. We have previously demonstrated that Max-null ESCs in the $2 \mathrm{i}+$ leukaemia inhibitory factor (LIF) condition with two kinase inhibitors against MEK and GSK3 $\beta$ (ref. 31), but not in conventional ESC medium, maintain normal expression levels of pluripotency markers such as OCT3/4, SOX2 and NANOG, and are able to self-renew indefinitely ${ }^{16}$. In this context, we sought to determine whether Max depletion leads to the activation of germ cell-related genes in ESCs even if the vast majority of cells maintain their pluripotent properties. First, DNA microarray analyses showed that the expression levels of germ cell-related genes were elevated even in Dox-treated Max-null ESCs under the $2 \mathrm{i}+\mathrm{LIF}$ condition, although the induction levels were not as conspicuous as those observed in Max-null ESCs under conventional LIF + serum conditions (Fig. 5a). Quantitative PCR confirmed the induction of Stra8 gene expression in both conditions (Fig. 5b). These results prompted us to investigate whether the cytological changes reminiscent of meiotic cell division also occur in Dox-treated Max-null ESCs under the $2 \mathrm{i}+\mathrm{LIF}$ condition. We found that accumulation of STRA8 protein as well as meiosis-like SYCP3 staining became evident on loss of Max expression in ESCs under the $2 \mathrm{i}+$ LIF condition (Fig. $5 c, d$ ), although the frequency of these cytological changes was about half of that observed in conventional ESC culture conditions (Fig. 5e).

Implications of direct onset of meiosis in Max-null ESCs. It has been shown that the $2 \mathrm{i}+$ LIF condition renders ESCs refractory to cellular differentiation ${ }^{32}$. The changes in meiosis reported in Max-null ESCs, even in the $2 \mathrm{i}+$ LIF condition, indicate that this induction occurs without passing through a PGC state that first becomes evident at around 7.0 days post coitum (dpc; early gastrulation stage) in mouse embryos ${ }^{33}$. This is supported by the fact that Max ablation in ESCs led to elevated expression levels of late PGC and meiosis-related genes, but did not elicit prominent induction of PGC specification genes. To further address this issue, and taking into account the fact that Blimp1 is known to play crucial roles in the induction and maintenance of PGCs ${ }^{34,35}$, we knocked out the Blimp1 gene in Max-null ESCs using the CRISPR-Cas9 system. After homozygous knockout of the Blimp1 gene (Supplementary Fig. 8), the ESCs were treated with Dox to suppress Max expression for 9 days and then subjected to co-immunocytochemical analyses with antibodies against SYCP3 and STRA8. Strong induction of STRA8 as well as meiosis-like SYCP3-staining patterns became evident in the ESCs after loss of Max expression, even without a functional Blimp1 gene (Fig. 5f-h), indicating that Max-null ESCs bypassed the PGC state and directly acquired features reminiscent of meiosis I phase.

Meiosis-related changes in ESCs depend on retinoid. Retinoid $^{7-10}$ and LIF ${ }^{36}$ signalling are critical players in the onset of meiosis in germ cells. We thus decided to examine the effects of RA and LIF on Max-null ESCs. Similarly to vitamin C, RA treatment further enhanced the meiosis-related changes in Max-null ESCs (Fig. 6a-c). With respect to LIF, although its withdrawal from culture showed little to no effect on Stra8 expression, it facilitated meiosis-related cytological changes in Max-null background (Fig. 6c). We also found that the number of viable cells was inversely correlated with the magnitude of meiosis-like changes (Fig. 6d), again implying that meiosis-like changes in ESCs are accompanied by apoptotic cell death. Our analyses with the RA receptor inhibitor AGN193109 (AGN; Fig. 6e and Supplementary Fig. 9a,b) and forced expression of Cyp26b1 encoding an RA-degrading enzyme (Fig. 6f, Supplementary Fig. 9c,d) also confirmed the crucial involvement of RA, likely present in fetal bovine serum used, in meiotic changes in Max expression-ablated ESCs. To confirm this, we conducted experiments using serum- and RA-free N2B27 medium. Without exogenous supply of RA, induction of Stra8 was barely detectable, irrespective of the presence or absence of Max expression in N2B27 medium. Interestingly, RA supplementation augmented Stra8 expression levels dosedependently even in the presence of Max expression, although much more profound induction was attained in the absence of Max expression. These results suggest that RA is essential for the induction of Stra8 expression and that Max expression-ablated background sensitized ESCs to the RA-mediated change (Fig. 6g).

Blockade of meiosis by MAX/MGA-containing atypical PRC1. To explore the molecular mechanisms underlying the meiosis-related changes associated with Max depletion in ESCs, we first searched for factors in the MYC superfamily, whose deficiency caused meiosis-related changes in ESCs, similar to MAX ablation. We found that lentivirus-mediated knockdown of Mga and Max, but not those of other MYC superfamily members, were coupled with induction of the expression of meiotic genes Stra8, Taf7l and Slc25a31 (Fig. 7a and Supplementary Fig. 10). Mga knockdown-mediated induction of STRA8 expression was also confirmed by immunostaining (Fig. 7b). Because both MAX and MGA were recently demonstrated to be a part of an atypical PRC1 complex (PRC1.6) ${ }^{14,15}$, we next examined the consequence of forced reduction of the expression of the $L 3 m b t l 2$ gene that 
a

Stra8 locus

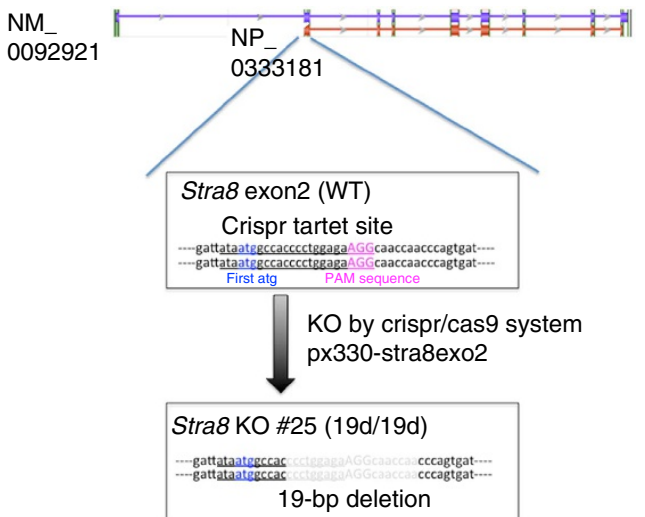

b
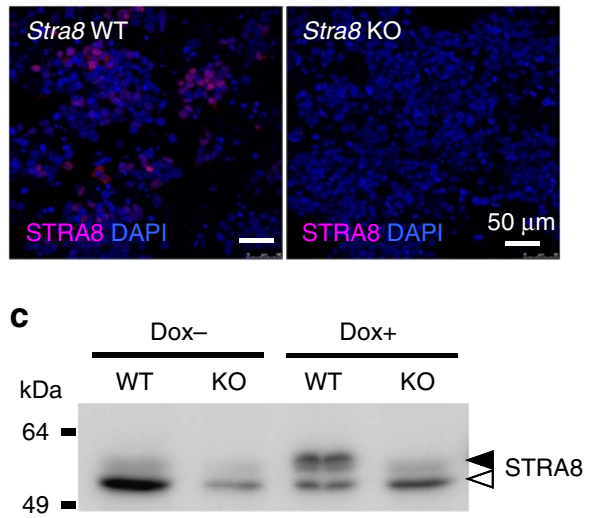

d

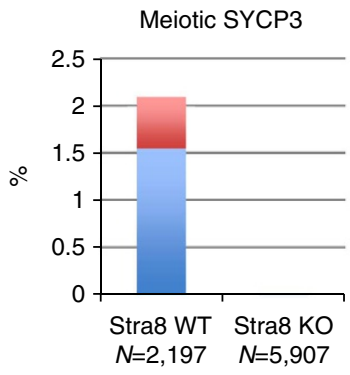

e

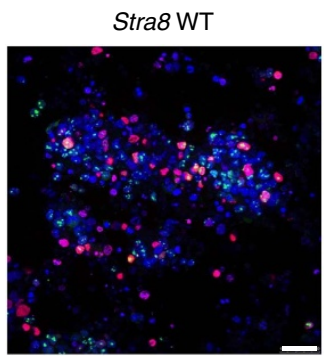

$\gamma$ H2AX SYCP3 DAPI

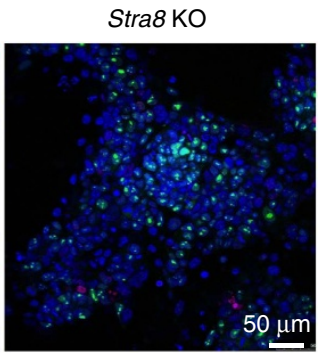

$\gamma$ H2AX SYCP3 DAPI f

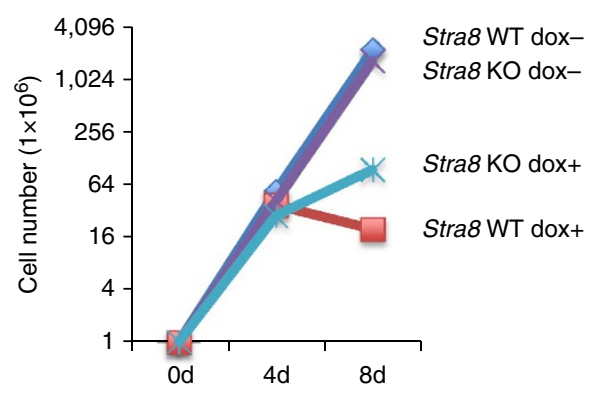

g

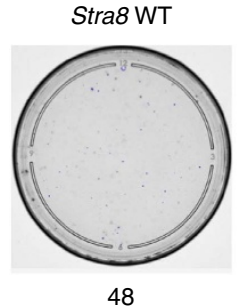

48
Stra8-KO (\#25)

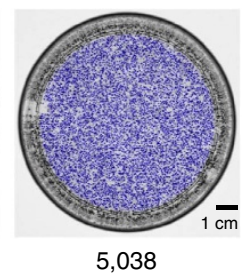

Figure 4 | Induction of the Max expression ablation-mediated meiosis-like SYCP3-staining pattern in ESCs depends on the presence of a functional Stra8 gene. (a) Schematic representation of CRISPR-Cas9-mediated disruption of the Stra8 gene. Genomic analyses revealed that Stra8 KO \#25 clone had an out-of-frame mutation in both loci of the Stra8 gene due to homozygous deletion of 19 base pairs located immediately downstream of the ATG initiation codon in exon 2 of the gene. (b) Confirmation of the absence of functional Stra8 genes in the Stra8 KO \#25 Max-null ESC clone. The Stra8 KO \#25 clone and parental Max-null ESCs were treated with Dox for 9 days and then subjected to immunocytochemical analyses with an antibody against STRA8. (c) Western blot analyses of STRA8 in Stra8 KO \#25 clone and parental Max-null ESCs cultured in the presence or absence of Dox. Solid and open arrowheads indicate specific and non-specific bands, respectively. (d) Frequency of preleptotene-, leptotene- and zygotene-like staining patterns in the Stra8 KO \#25 clone and parental Max-null ESCs treated with Dox for 9 days. (e) Immunostaining analyses of $\gamma \mathrm{H} 2 \mathrm{AX}$ and SYCP3 in the Dox-treated (9 days) Stra8 KO \#25 clone and parental Max-null ESCs. (f) Increase in the recovery of viable Dox-treated Max-null ESCs due to homozygous knockout of the Stra8 gene. The number of cells at day 0 was arbitrarily set to one. $(\mathbf{g})$ Max-null ESCs $\left(1 \times 10^{5}\right)$ with or without functional Stra8 genes were individually transferred to $10-\mathrm{cm}$ dishes and treated with Dox for 12 days. The cells were then subjected to Leishman's staining. Numbers under the dishes are the number of stained cell colonies. d, day; KO, knockout; WT, wild type.

also encodes one of the components of PRC1.6. Knockdown of L3mbtl2 expression also led to significant elevation in the expression levels of meiotic genes, although the expression levels of the Stra8 gene were not affected (Fig. 7c). Next, to assess whether elevation of meiotic gene expression is the direct consequence of Max expression ablation or secondary in nature, we examined whether MAX protein binds to the meiosis-related genes. To this end, we conducted chromatin immunoprecipitation (ChIP) analyses with an anti-MAX antibody. Our data clearly demonstrated that MAX bound to Ddx4, Slc25a31 and Sycp3 genes in Dox-untreated ESCs (Fig. 7d).
However, such signals were attenuated on Max expression ablation by Dox treatment. These results indicated that the MAX-containing complex directly bound to meiosis-related genes and repressed their expression in ESCs, and upregulation of these genes in Max-null ESCs reflects the liberation from such MAX-dependent repression. We also conducted ChIP analyses with an antibody against L3MBTL2. Although the immunoprecipitation efficiency was not as high with the antibody against L3MBTL2 compared with the anti-MAX antibody, our data indicated that L3MBTL2 directly bound to the examined meiotic genes. Furthermore, our data demonstrated that the 
a

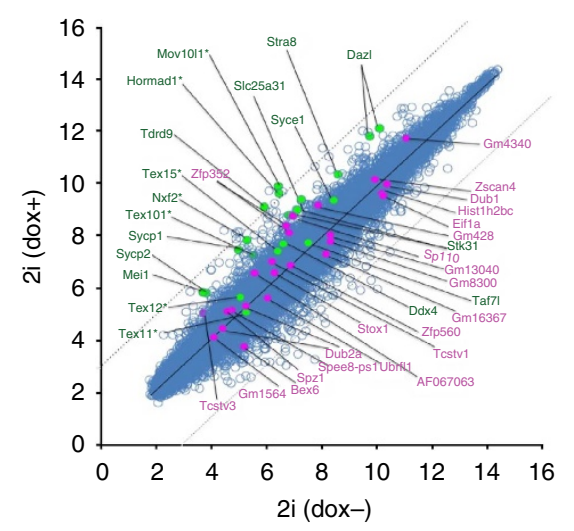

C
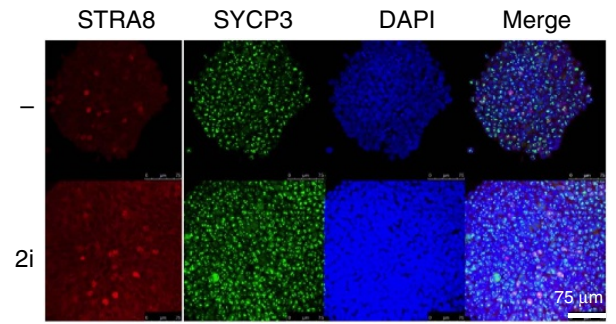

e

Meiotic SYCP3

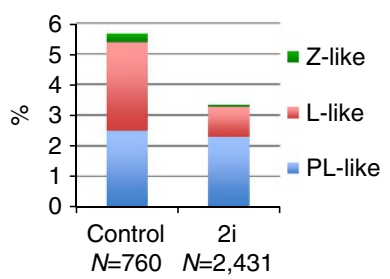

g

Blimp1 KO \#6

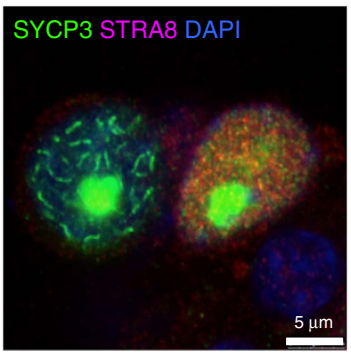

f

h b

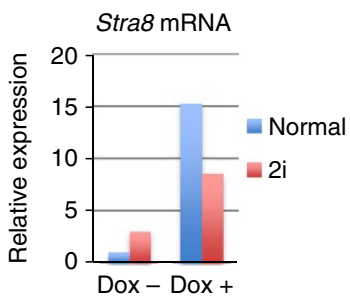

d
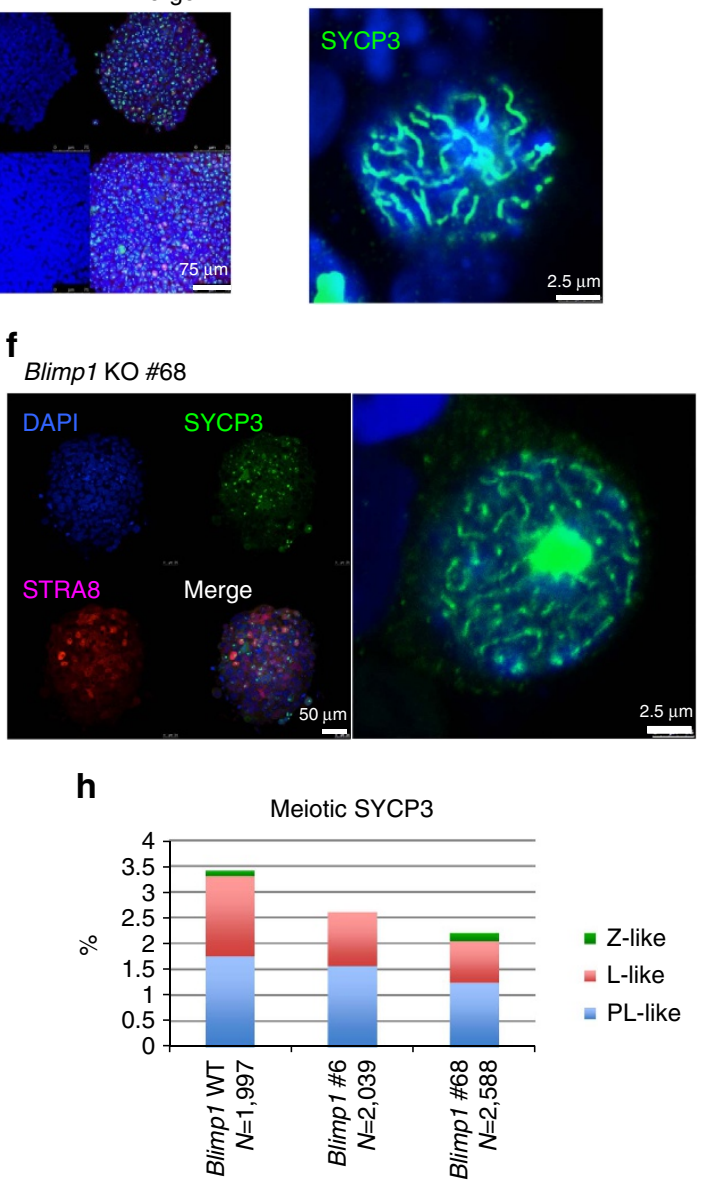

Figure 5 | Occurrence of meiosis-like changes in Max expression-ablated ESCs without passing through the PGC state. (a) Scatter plots of DNA microarray data from untreated and Dox-treated Max-null ESCs cultured under the $2 i+$ LIF condition. Genes highlighted in Fig. 1a are indicated by the same colours (green: germ cell-specific genes; pink: two-cell embryo-specific genes; green with an asterisk: genes belonging to both categories). (b) Measurement of Stra 8 mRNA levels by quantitative PCR in Dox-untreated and -treated Max-null ESCs cultured under conventional mouse ESC or $2 i+$ LIF culture conditions. (c) Immunocytochemical analyses of STRA8 and SYCP3 in Dox-treated Max-null ESCs cultured under conventional mouse ESC or $2 \mathrm{i}+$ LIF culture conditions. (d) Representative image of the zygotene-like SYCP3-staining pattern observed in Dox-treated Max-null ESCs cultured under the $2 i+$ LIF condition. (e) Frequency of meiosis-like SYCP3-staining patterns in Dox-treated Max-null ESCs cultured under conventional mouse ESC or $2 \mathrm{i}+$ LIF culture conditions. (f) Immunocytochemical analyses of STRA8 and SYCP3 in the Dox-treated Blimp1 KO \#68 Max-null ESC clone. Right panel shows a representative zygotene-like SYCP3-staining pattern. In this mutant clone, a single adenine nucleotide was inserted into both loci of exon 3 in the Blimp1 gene, causing an out-of-frame mutation (for details, see Supplementary Fig. 8). (g) Leptotene-like immunostaining pattern of SYCP3 with high STRA8 expression (right) and the zygotene-like SYCP3-staining pattern (left) with low STRA8 expression in the Dox-treated Blimp1 KO \#6 Max-null ESC clone in which a large deletion had occurred in the region encompassing exon 3 and intron 3 in both loci of the gene (for details, see Supplementary Fig. 8). (h) Frequency of meiosis-like SYCP3-staining patterns in Dox-treated Max-null ESCs of Blimp1 KO \#68 and \#6 clones. KO, knockout. 
a

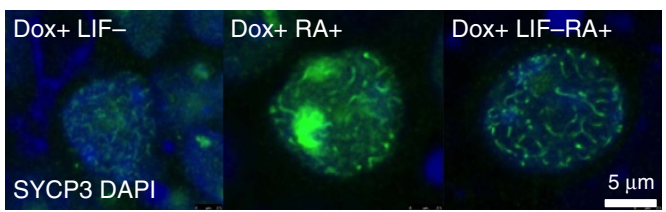

6 days culture b

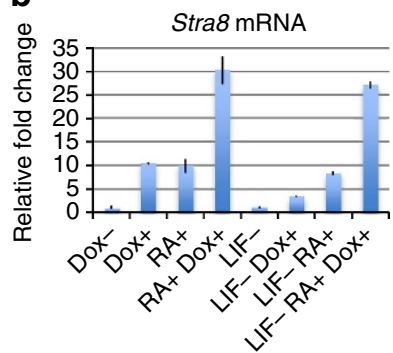

d

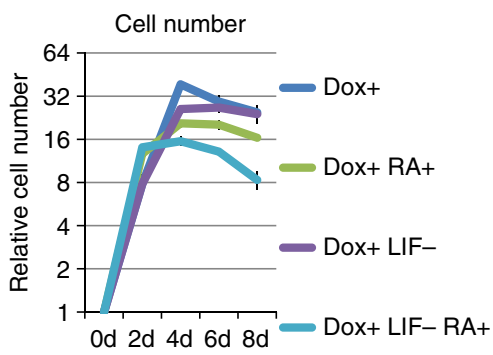

f

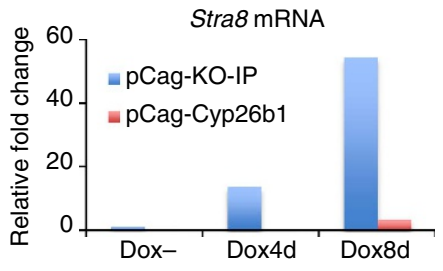

g
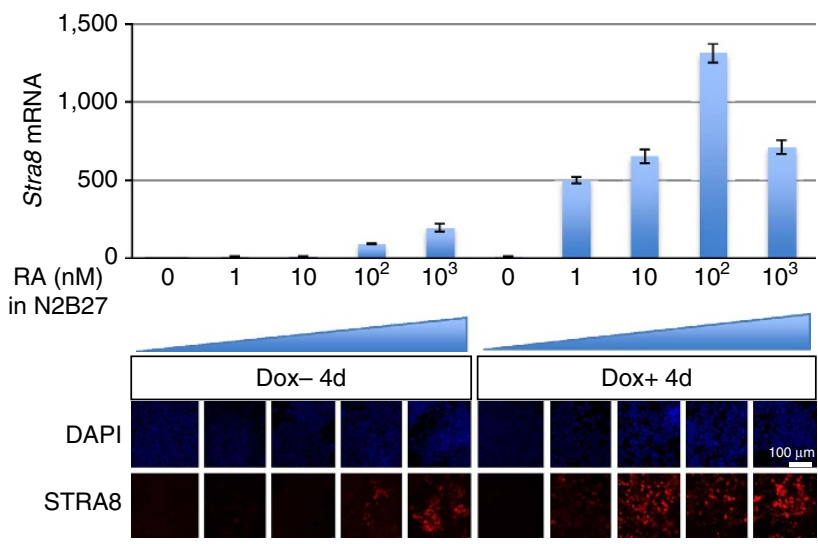

Figure 6 | RA treatment potentiates meiosis-related changes in Max expression-ablated ESCs. (a) Representative images of meiosis-like SYCP3-staining patterns in Dox-treated Max-null ESCs cultured with or without RA in the presence or absence of LIF for 6 days. (b) Expression levels of the Stra8 gene in Dox-treated Max-null ESCs cultured under the indicated conditions for 6 days. The Stra8 gene expression level in Dox-untreated control Max-null ESCs cultured in the presence of LIF was arbitrarily set to one $(n=3$, means \pm s.d.). (c) Frequency of meiosis-like SYCP3-staining patterns in Max-null ESCs treated with Dox for 6 days or together with RA in the presence or absence of LIF. (d) Effect of RA on the recovery of viable Dox-treated Max-null ESCs cultured with or without LIF. The number of cells at day 0 was arbitrarily set to one $(n=3$, means \pm s.d.). (e) Quantification of Stra8 mRNA levels in Dox-treated or -untreated Max-null ESCs cultured in the presence or absence of the RA receptor inhibitor AGN for the indicated days ( $n=3$, means \pm s.d.). (f) Effect of the expression of Cyp26b1 on Stra8 expression levels in Max-null ESCs. (g) Dose-dependent effect of RA on Stra8 expression levels in Max-null ESCs cultured in N2B27 with no RA as basal medium ( $n=3$, means \pm s.d.).

binding signal was greatly diminished on Max expression ablation, implying that binding of L3MBTL2 on the meiotic genes is dependent on the presence of MAX (Fig. 7d). This finding further supported the notion that PRC1.6 complex is involved in the repression of meiotic genes in ESCs. Next, we examined the expression levels of genes encoding the $M y c$ superfamily and those encoding either one of the components of the PRC1.6 complex after Max expression ablation in ESCs. We found that many of the genes, including $L 3 \mathrm{mbtl} 2$, showed slight decline in their expression levels following Max depletion in ESCs, while expression level of $M g a$ was initially elevated (up to 4 days) but declined thereafter (Supplementary Fig. 11). 
a

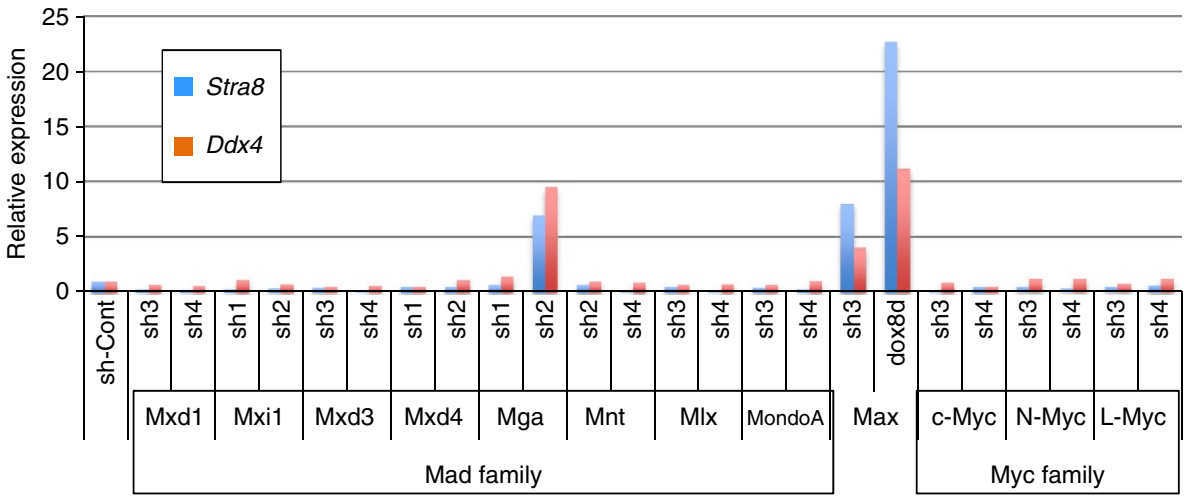

b

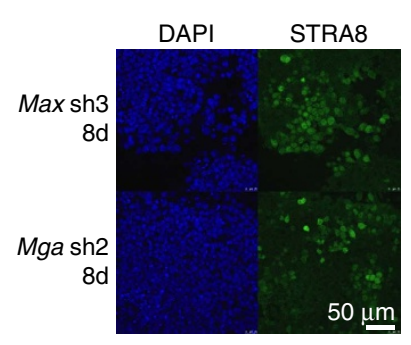

C

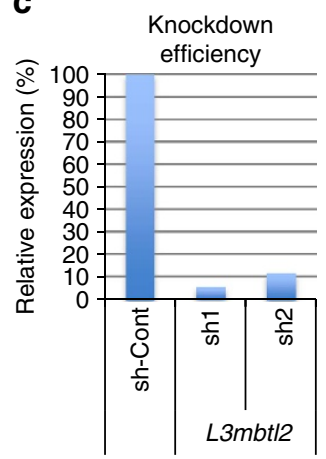

SIc25a31 TSS

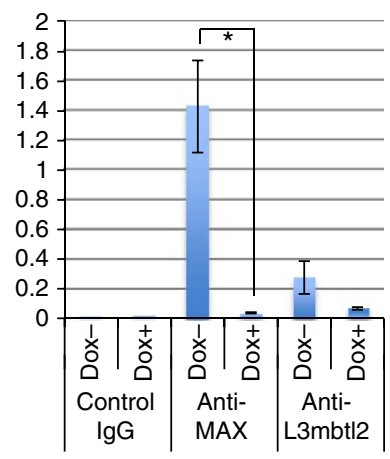

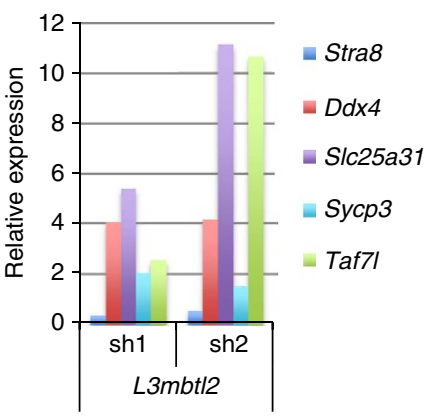

Sycp3 TSS

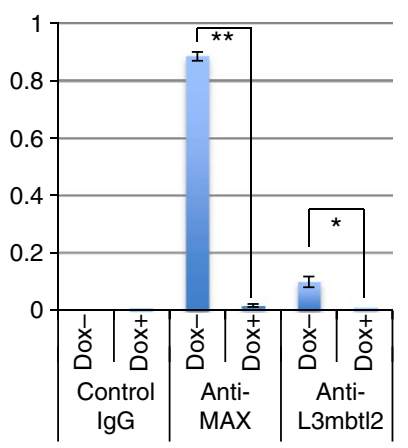

Figure 7 | Implication of the MAX-containing PRC1.6 complex in the blockade of meiosis-related gene expression in ESCs. (a) Effect of knockdown of Myc superfamily genes on the expression of Stra8 and Ddx4 genes in ESCs. Expression levels of Stra8 and Ddx4 genes were quantitated in Dox-untreated Max-null ESCs subjected to lentivirus-mediated stable knockdown of the indicated genes. Expression levels of Stra8 and Ddx4 genes in cells subjected to knockdown with a scrambled sequence was arbitrarily set to one. Knockdown efficiency of each gene is shown in Supplementary Fig. 10a.

(b) Immunocytochemical analyses of STRA8 in Dox-untreated Max-null ESCs in which expression of Max (upper) and Mga (lower) was subjected to lentivirus-mediated stable knockdown. (c) Effect of L3mbt/2 knockdown on meiotic gene expression in ESCs. Lentivirus-mediated knockdown was conducted as described in a. Expression of Stra8, Ddx4, S/c25a31, Sycp3 and Tafl7 was determined by TaqMan-based quantitative PCR. (d) ChIP analyses of meiotic gene promoters with antibodies against MAX and L3MBLT2. Dox-untreated and -treated Max-null ESCs for 4 days were subjected to ChIP with either anti-MAX or L3MBLT2 antibodies. A ChIP reaction was also performed with control IgG. The genomic DNAs recovered by these ChIP reactions were individually subjected to quantitative PCR to quantify the relative amounts of Ddx4, S/c25a31 and Sycp3 gene promoters compared with those used as input samples $(n=3$, means \pm s.d.). Unpaired $t$-test was done to compare immunoprecipitation efficiency statistically between Dox-untreated and -treated Max-null ESCs $\left({ }^{\star} P<0.05 ;{ }^{\star \star} P<0.01\right)$.

Meiosis-like changes in ESCs with a MYC/MAX complex. We have previously demonstrated that the detrimental phenotype of Max-null ESCs could be rescued by c-MYC and MAX mutants bearing modifications in their leucine zipper dimerization domains $^{16,36}$. Because of these modifications, c-MYC and MAX mutants, designated as c-MYC-EG and MAX-EG, respectively, do not bind to other endogenous partners, but dimerize efficiently with each other to function as a c-MYC/MAX transcriptional complex. Therefore, Max-null ESCs bearing these mutants are able to maintain MYC activity but lack a functional PRC1.6 complex because the MAX-EG mutant does not interact with the endogenous MGA protein. We examined whether meiosis-like changes were evident in Max-null ESCs expressing c-MYC-EG and MAX-EG. Consistent with our previous report, Max-null ESCs bearing these mutants were able to maintain cell viability (Fig. 8a,b). However, our analyses revealed that the induction of expression of meiotic genes and production of STRA8 protein were also observed in these cells (Fig. 8c,d). Furthermore, 
a

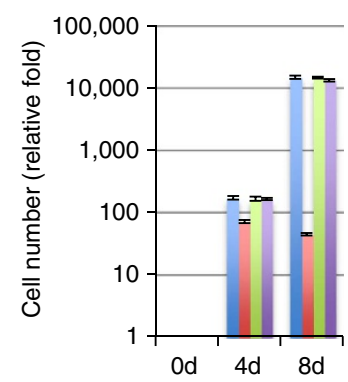

C

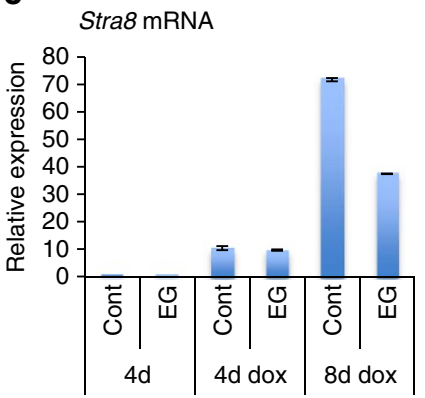

- Control dox-

- Control dox+

EG Dox-

- EG Dox+

b
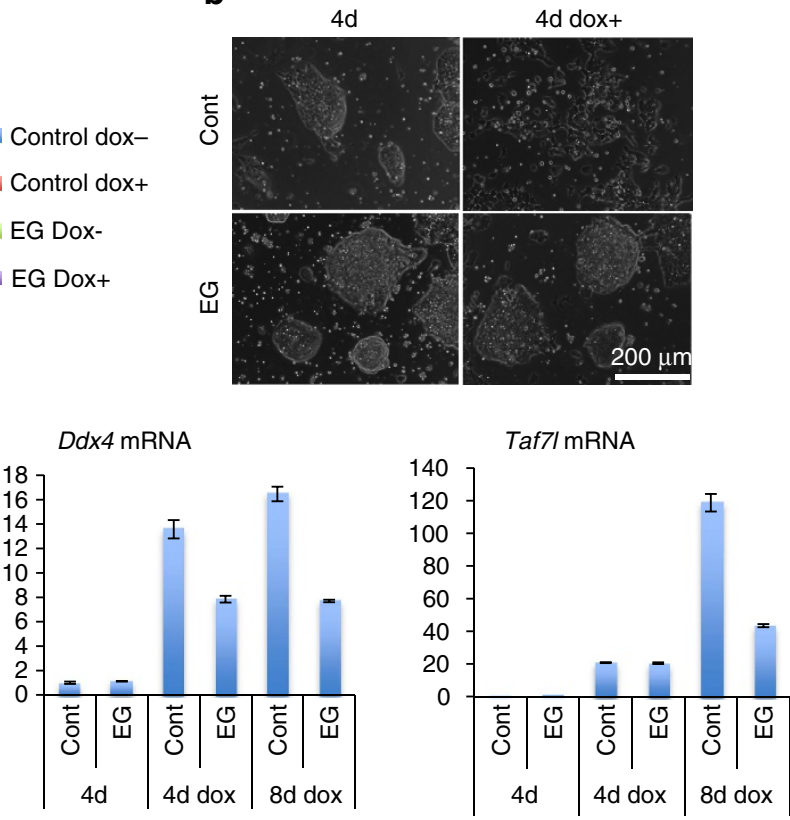

d

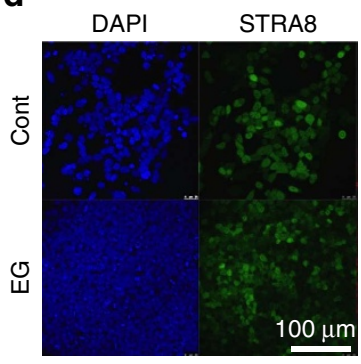

e

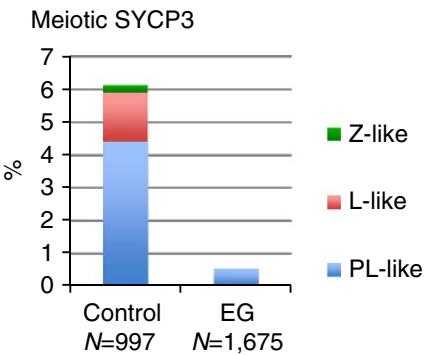

Figure 8 | Meiosis-like changes in Dox-treated Max-null ESCs with rescued viability by mutant c-MYC/MAX. (a) Max-null ESCs with or without mutant $\mathrm{c}-\mathrm{MYC} / \mathrm{MAX}$, whose expression was under the control of the constitutive $\beta$-actin promoter, were treated with Dox for the indicated days, and then viable cell numbers were counted. The number of cells at day $0(\mathrm{Od})$ was arbitrarily set to one $(n=3$, means \pm s.d.). (b) Microscopic inspection of Dox-treated and -untreated Max-null ESCs for 4 days (4d) with or without mutant c-MYC/MAX. (c) Examination of the expression levels of meiotic genes in Doxtreated and untreated Max-null ESCs with or without mutant c-MYC/MAX. Expression levels of Stra8, Ddx4 and Tafl7l were examined by TaqMan-based quantitative PCR ( $n=3$, means \pm s.d.). (d) Max-null ESCs with or without mutant c-MYC/MAX were treated with Dox for 8 days ( $8 d$ ) and then subjected to immunostaining with antibodies against STRA8. (e) Frequency of the meiosis-like SYCP3-staining pattern in Dox-treated Max-null ESCs with or without mutant c-MYC/MAX for 8 days. Cont, control.

meiosis-like cytological changes were detected as well (Fig. 8e). These meiotic-like changes were found, however, to a lesser degree when compared with control Max-null ESCs. These results suggest that a PRC1.6-independent MYC/MAX activity may also help guard meiosis entry in ESCs.

In vivo meiosis is coupled with a decline in Max expression. Since meiosis-like cytological changes could be induced in Max expression-ablated ESCs, we decided to examine whether Max expression levels decline during normal meiotic cell division in testes. To this end we performed immunohistochemical analyses of testis sections. In addition to an antibody against MAX, antibodies against PLZF and cKIT, which mark spermatogonial stem/progenitor cells and differentiating spermatogonia, respectively, were used in the analyses to facilitate staging of cells undergoing meiosis ${ }^{37}$. We found that MAX expression, which is generally thought to be ubiquitous ${ }^{38}$, was extremely low in cKIT-positive differentiating spermatogonia and premeiotic type B spermatogonia at stage VI compared with PLZF-positive spermatogonial stem/progenitor cells (Fig. 9a-c).
This low level of MAX expression in differentiating spermatogonia persisted up to the early pachytene stage but increased again during the pachytene stage (Supplementary Fig. 12). Whereas the meiotic cell division begins in testes after birth in males, meiosis commences in the genital ridges at the midgestation stage in females ${ }^{39}$. Therefore, we examined whether the meiotic processes in female mice are also accompanied by downregulation of MAX expression. Indeed, we found that MAX expression levels were extremely low in SYCP3-positive female PGCs at $13.5 \mathrm{dpc}$, which coincides with meiosis initiation, but were much higher in E13.5 male PGCs (Fig. 9d). These results reveal that, although occurs at different timing, meiosis in both sexes is coupled with downregulation of MAX expression. To assess whether there is also a difference in Max mRNA levels between male and female PGCs, we inspected DNA microarray data of PGCs deposited by Kagiwada et al. ${ }^{40}$ We found that Max expression levels in female PGCs was lower compared with those of male PGCs, especially at the initiation of meiosis (13.5 dpc; Supplementary Fig. 13a), implicating that the lower level of MAX protein in female PGCs revealed by immunostaining was at least in part due to lower levels of Max mRNA in female PGCs 
a
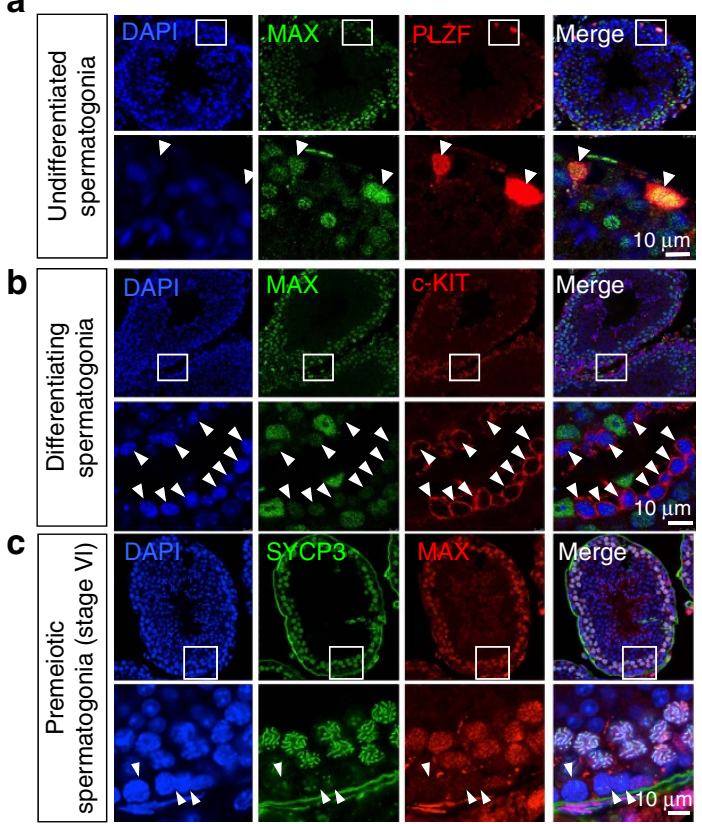

d
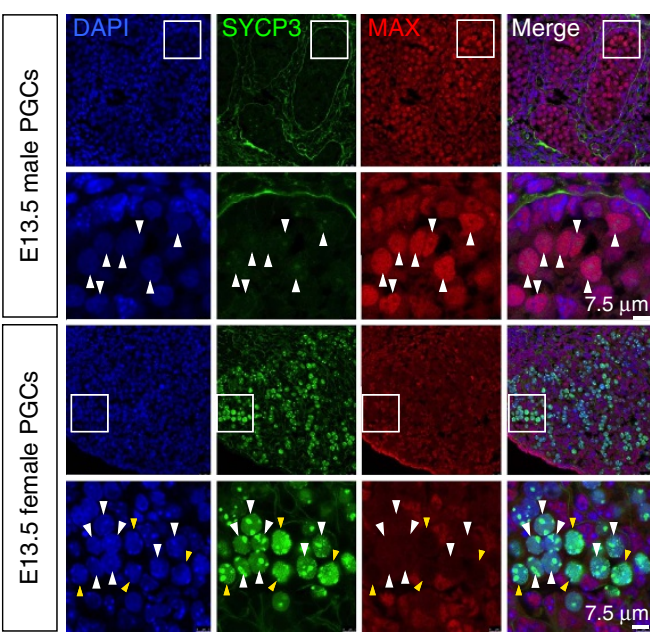

Figure 9 | Max expression dynamics in normal gametogenesis. Frozen sections of testes from adult male mice were prepared and immunostained for MAX. The sections were also co-immunostained for PLZF (a), CKIT (b) or SYCP3 (c). Arrowheads in $\mathbf{a}, \mathbf{b}$ and $\mathbf{c}$ indicate PLZF-positive undifferentiated spermatogonia that are strongly positive for MAX, cKITpositive differentiating spermatogonia that are weak for MAX and cells undergoing meiotic cell division (presumable premeiotic type $B$ spermatogonia) that are weak for both SYCP3 and MAX, respectively. (d) Decline of MAX expression levels in female, but not in male, PGCs of 13.5 $\mathrm{dpc}$ embryos. Male and female embryos (13.5 dpc) were recovered from a single pregnant female mouse, and then the genital ridges were recovered individually. After processing, sections were prepared and subjected to co-immunostaining with antibodies against MAX and SYCP3. Arrowheads shown in male genital ridges represent PGCs with strong and weak signals for MAX and SYCP3, respectively. Corresponding female PGCs with strong expression of SYCP3 are also indicated by arrowheads in which white and yellow indicate weak and almost no MAX expression, respectively.

compared with that in male PGCs. However, our data do not exclude the possibility that MAX protein levels were also controlled post-translationally.

We also used these DNA microarray data to study the expression dynamics of genes whose expression levels were elevated in Max-null ESCs. Both germline-specific genes and those categorized into both germline and two-cell signature genes shown in Supplementary Fig. 1 also had strong elevations in their expression levels during midgestation (E10.5-13.5; Supplementary Fig. 13b). Interestingly, most meiotic genes showed significant elevations in their expression levels not only in female PGCs but also in male PGCs during gestation, although the magnitude of the elevation was not as prominent as that observed in female PGCs. More remarkably, most of the genes that were significantly or modestly activated in Max-null ESCs were also, respectively, activated in similar manners during PGCs development, providing additional evidence that our Max expression ablation system in ESCs represents a bona fide model of meiosis. However, genes categorized exclusively as two-cell signature genes did not show any tendency of activation, but rather decreased their expression levels during PGCs development. These results further suggest that activation of these genes in Max expression-ablated ESCs is not related to meiosis-like changes (Supplementary Fig. 13b).

Meiotic induction in GSCs by Max knockdown. We next examined the consequence of forced reduction of Max expression in GSCs. We not only found that Stra8 expression level was elevated (Fig. 10a) but also detected cytological changes reminiscent of meiosis in GSCs on Max knockdown (Fig. 10b,c). Of note is that these cytological changes were only observed in cells with reduced Max expression. Because knockdown of Max expression was insufficient, but Max expression should be completely ablated in ESCs to induce meiosis-related cytological changes, our data with GSCs suggested that GSCs are more competent for meiosis-like cytological changes than ESCs. Next, we conducted knockdown of Max expression in mouse embryonic fibroblasts, NIH3T3 cells and induced pluripotent stem cells (iPSCs). Although lentivirus-mediated knockdown of Max expression enhanced Stra 8 gene expression levels in iPSCs, no such induction was evident in mouse embryonic fibroblasts or NIH3T3 cells (Fig. 10d). Induction of STRA8 in iPSCs was confirmed by immunostaining (Fig. 10e). Therefore, we conclude that meiosis-related changes due to Max expression deficiency are specific to germ and pluripotent cells.

\section{Discussion}

In this study we have uncovered an intriguing meiosis-like programme in ESCs triggered by loss of function of Max. Our results show that the loss of Max expression in ESCs not only activates germ cell-related genes but also leads to cytological changes resembling leptotene and zygotene stages of meiosis.

Our functional screening of the $M y c$ superfamily led to the identification of MGA, whose absence also triggered the meiosis-like changes. Our subsequent analyses revealed that a MAX-containing atypical PRC1 complex (PRC1.6) is responsible for the molecular blockade of meiotic onset. There are six distinct PRC1 complexes with variations in their components, but only in PRC1.6 (ref. 14) are both MAX and MGA present. A unique characteristic of PRC1.6 is that this variant PRC1 does not co-localize with PRC2 at their target sites ${ }^{15}$. Therefore, PRC1.6 must bind to DNA stably without PRC2, and possible molecules responsible for such stable DNA binding include MAX and its association factor MGA. In this context, it is of interest to mention that the MAX-containing PRC1 complex has been shown to be linked to the $\mathrm{E}$ box sequence that serves as a binding site for MAX and MGA ${ }^{15}$.

We provide evidence of the existence of a transient decline in Max expression during physiological gametogenesis in both male and female germ cells. This lead us to assume that the same or at least a similar process of MAX-dependent blockade of meiosis is 
a
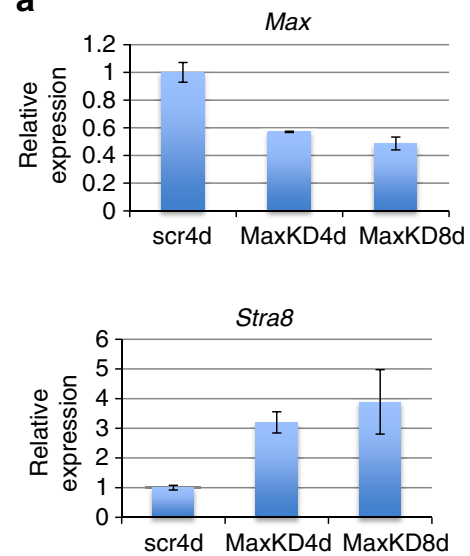

b

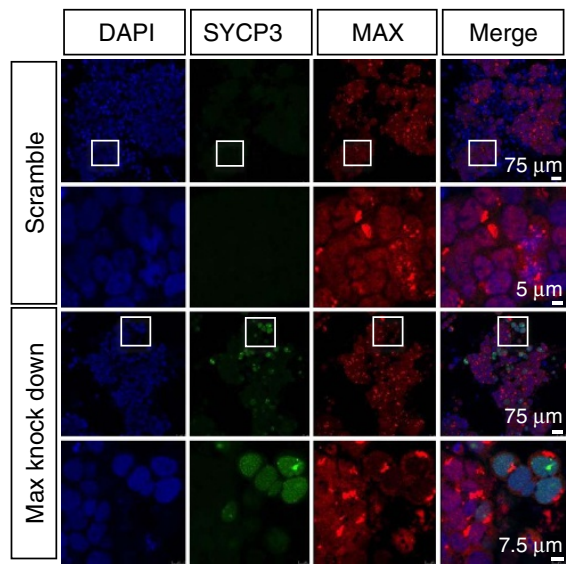

e

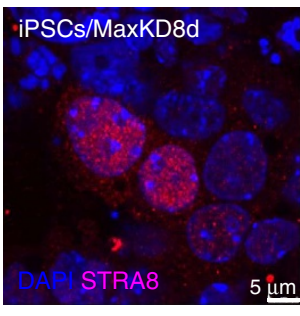

$5 \mu \mathrm{n}$

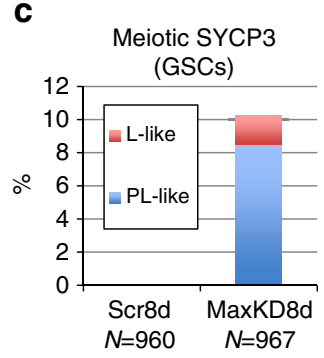

d

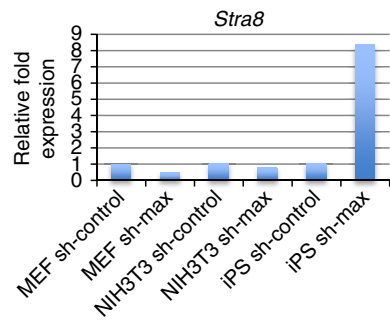

f

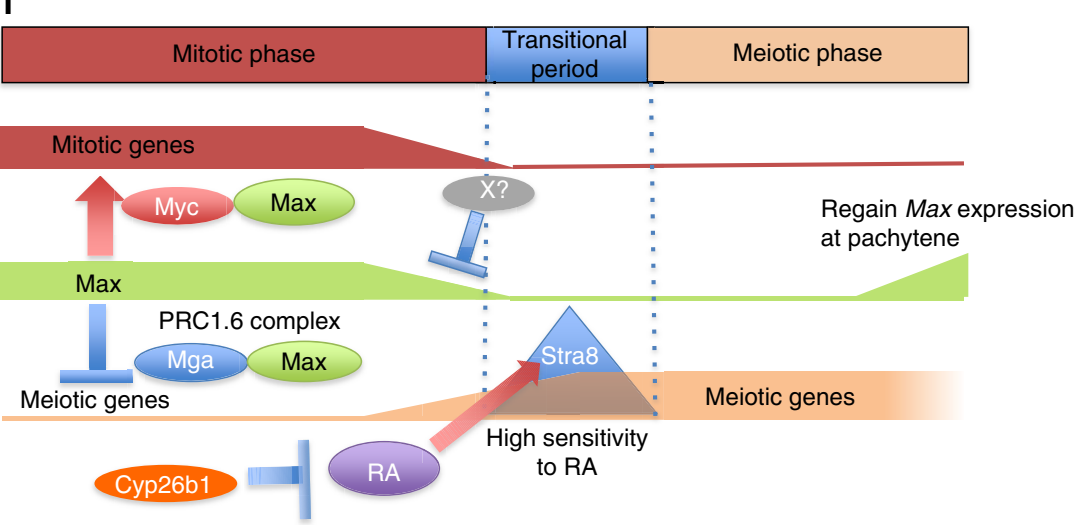

Figure 10 | Induction of meiosis-like changes in GSCs by knockdown of Max expression. (a) Effect of Max knockdown in GSCs on expression of the Stra8 gene. Lentivirus-mediated knockdown was conducted as described in Fig. 7. Upper panel shows the knockdown efficiency of Max expression ( $n=3$, means \pm s.d.). (b) Effect of Max knockdown in GSCs on induction of the meiosis-like SYCP3-staining pattern. GSCs were subjected to knockdown with scrambled or specific sequences for Max expression and then applied to co-immunostaining analyses with antibodies against MAX and SYCP3. (c) Frequency of the meiosis-like SYCP3-staining pattern in GSCs subjected to lentivirus-mediated knockdown of Max expression. (d) Effect of Max knockdown in MEFs, NIH3T3 cells and iPSCs on the induction of Stra8 expression. The levels of Stra8 expression were measured by quantitative PCR.

(e) Immunocytochemical analyses of iPSCs subjected to lentivirus-mediated knockdown of Max expression with an antibody against STRA8. (f) Schematic representation of possible inverse regulation of the expression of meiotic and mitotic genes through the control of Max expression for efficient meiosis. MAX is known to play important roles in preserving the stem cell properties of ESCs and GSCs by functioning as an obligated partner for MYC transcription factors. Our present study demonstrates that MAX also contributes to the maintenance of stemness of these cells by blocking expression of meiotic genes. Artificial and physiological declines in Max expression levels in ESCs and GSCs, respectively, enhance and reduce expression of meiotic and mitotic genes, respectively, through liberation from the MAX/MGA-dependent repression mechanism and the loss of MYC/MAX complex-dependent transcriptional activation. The former potentiates meiotic progression, whereas the latter results in disruption of the stem/progenitor properties and, therefore, facilitates cellular differentiation. Our data suggest that the MAX-containing PRC1.6 complex is the molecule responsible for the MAX-mediated repression of meiotic genes. However, it is unknown at present what lowers Max expression levels at the onset of meiosis in GSCs.

present in GSCs. GSCs should be liberated from such blockades by a physiological decline in Max expression to proceed with meiosis during their differentiation. This assumption was further corroborated by data demonstrating that knockdown of Max expression in GSCs also led to meiosis-like cytological changes. It is noteworthy that MYC family proteins, including c-MYC and $\mathrm{N}-\mathrm{MYC}$, play crucial roles in preservation of the self-renewal properties of both $\mathrm{ESCs}^{41}$ and $\mathrm{GSCs}^{42}$ in which MAX functions as an indispensable partner. Thus, as depicted in Fig. 10f, loss of MAX may promote meiosis not only by activating the expression 
of meiotic genes but also by facilitating cellular differentiation through impairment of the self-renewal of ESCs and GSCs because of the loss of MYC functions. The differences in efficiencies in the induction of meiosis-like changes between Max-null ESCs bearing c-MYC, MAX mutants and control Max-null ESCs may be explained by the preservation of MYC functions in the former, but not in the latter cells. It is noteworthy that Yokobayashi et al. ${ }^{43}$ have demonstrated that the PRC1 complex acts negatively against RA signalling through maintenance of the transcriptionally repressed state of the Stra 8 gene. Similarly, our data demonstrated that Max expression ablation in ESCs, which is coupled to disruption of the PRC1.6 complex, sensitized RA-mediated induction of Stras gene expression. This finding further strengthens the notion that the meiotic onset in ESCs faithfully recapitulates the in vivo meiotic onset. Observations made by Yokobayashi et al. ${ }^{43}$ on the loss of function of PRC1 in germ cells may not reflect impairment of the general functions of PRC1, but might be due to disruption of the MAX-containing atypical PRC1 complex.

Max expression ablation appears to induce meiotic onset in ESCs in a physiologically relevant manner. However, such meiosis-like cells are arrested at rather early stages and do not proceed beyond the crux of the pachytene-like stage. To overcome this problem, development of improved culture conditions suitable for meiosis and/or the forced expression of inefficiently induced meiotic genes such as Rec8 and Sycp1 will be needed. Although we are far from a complete understanding of the mechanisms involved in generating haploid cells, the results and methodologies here described may open up new avenues towards achieving this goal.

\section{Methods}

Animal ethics. This study was carried out under strict accordance with the international and institutional guidelines. The protocol was approved by the institutional review boards on the Ethics of Animal Experiments of the Saitama Medical University, with the permission number of 1518.

Mouse ESC and GSC culture. Max-null ESCs with Max cDNA under the control of the tetracycline-off system were cultured in conventional ESC culture medium with LIF and serum, or the $2 \mathrm{i}$ condition with inhibitors of MEK (PD0325901) and GSK3 $\beta$ (CHIR99021) with the concentrations of 1 and $3 \mu \mathrm{M}$, respectively. N2B27 not containing vitamin A was used as basal medium for the experiments shown in Fig. $6 \mathrm{~g}$. The following reagents were used at the indicated concentration unless stated otherwise: $1 \mu \mathrm{g} \mathrm{ml}^{-1}$ Dox (631311, Clontech); $100 \mathrm{nM}$ VX680 (A10981-25, ADG); $20 \mu \mathrm{M}$ Z-VAD-FMK (627610, Calbiochem); $5 \mu \mathrm{M}$ AGN193109 (sc210768, Santa Cruz Biotechnology); $1 \mu \mathrm{M}$ all-trans retinoic acid (R2625, Sigma); and $50 \mu \mathrm{g} \mathrm{ml}^{-1}$ vitamin C (A4544, SIGMA). Mouse GSCs were cultured with Stem Pro-34 SFM medium (10639-011, Invitrogen) with the ingredients of $25 \mu \mathrm{g} \mathrm{ml}^{-1}$ insulin (1882, Sigma), $100 \mu \mathrm{g} \mathrm{ml}^{-1}$ transferrin (Sigma, T1147), $60 \mu \mathrm{M}$ putrescine (P7505, Sigma), $30 \mathrm{nM}$ sodium selenite $(214485$, Sigma $), 6 \mathrm{mg} \mathrm{ml}^{-1} \mathrm{D}-(+)$-glucose (G7021, Sigma), $30 \mu \mathrm{g} \mathrm{ml}^{-1}$ pyruvic acid (107360, Sigma), $1 \mu \mathrm{g} \mathrm{ml}{ }^{-1}$ DL-lactic acid (4263, Sigma), $5 \mathrm{mg} \mathrm{ml}^{-1}$ bovine serum albumin (15561-020, Invitrogen), $2 \mathrm{mM}$ L-glutamine, $50 \mathrm{nM}$ 2-mercaptoethanol, MEM vitamin solution (11120-052, Invitrogen), $0.1 \mathrm{mM}$ nonessential amino acid solution (11140-050, Invitrogen), $30 \mathrm{ng} \mathrm{ml}^{-1} \beta$-estradiol (E2758, Sigma), $60 \mathrm{ng} \mathrm{ml}^{-1}$ progesterone (P8783, Sigma), $10 \mu \mathrm{g} \mathrm{ml}^{-1}$ D-biotin (B4501, Sigma), $100 \mathrm{mM}$ ascorbic acid (A4544, Sigma), $20 \mathrm{ng} \mathrm{ml}^{-1}$ mouse epidermal growth factor (2028-EG, R\&D systems), $10 \mathrm{ng} \mathrm{ml}^{-1}$ human basic fibroblast growth factor (13256-029, Becton Dickinson),

$1,000 \mathrm{U} \mathrm{ml}^{-1}$ mouse leukaemia inhibitory factor (02740, Stem Cell Technologies), $10 \mathrm{ng} \mathrm{ml}^{-1}$ rat glial cell line-derived neurotrophic factor (512-GF, R\&D systems) and $1 \%$ fetal bovine serum.

Plasmid constructions. Construction of expression vectors for c-MYC and MAX mutants, designated as c-MYC-MG and MAX-EG, respectively, which do not bind to their endogenous partners, but bind efficiently with each other, was described previously ${ }^{16,36}$. CYP26B1 expression vector was constructed by PCR amplification using cDNA from mouse ESCs and subcloned into pCAG-IP vector bearing puromycin resistant gene.

Quantitative reverse transcription (RT)-PCR analysis. Quantitative RT-PCR (qRT-PCR) was performed using the StepOnePlusTM Real-Time PCR System (Applied Biosystems), with cDNAs generated by reverse transcription.
TaqMan-based reactions (Invitrogen) quantified the expression levels of Stra8 (Mm00486473_m1), Slc25a31 (Mm00617754_m1), Sycp3 (Mm00488519_m1), Taf7l (Mm00459354_m1), Ddx4 (Mm00802445_m1), c-Myc (Mm00487803_m1), N-Myc (Mm00476449_m1), L-Myc (Mm03053598_s1) and Max

(Mm00484802_g1), while qRT-PCR for other genes, including quantification of other $M y c$ superfamily genes such as $M x d 1$ (Supplementary Fig. 10), was done by SYBR Green-based method with the following oligonucleotides.

L3mbtl2: $5^{\prime}$-TACCTCATGAAGCGGTTGGT- $3^{\prime}$ and $5^{\prime}$-CAACCCACTGGGT GGATC- $3^{\prime}$

Mxd1: $5^{\prime}$-AGATGCCTTCAAACGGAGGAA- $3^{\prime}$ and $5^{\prime}$-CAAGCTCAGAGTG GTGTGTCG-3'

Mxi1: $5^{\prime}$-AACATGGCTACGCCTCATCG-3' and $5^{\prime}$-CGGTTCTTTTCCAA

CTCATTGTG-3

$M x d 3: 5^{\prime}$-GAGGCAGAGCACGGTTATG-3' ${ }^{\prime}$ and $5^{\prime}$-TGTAGTGTATCGGG

TACAGTCAA-3'

Mxd4: $5^{\prime}$-ATGGAGCTGAACTCTCTGCTG-3' and 5'-GTGAAGACCTGTTGT TCGGGG-3'

Mga: $5^{\prime}$-GAGGAGCACCTACCTACCTTCTTTGT- $3^{\prime}$ and $5^{\prime}$-ACGGGCAT

CTCGATTAGTAACT-3'

$M n t: 5^{\prime}$-TCCTGTAGTGACCAATTCCCC- $3^{\prime}$ and $5^{\prime}$-TGGCTCCTTAATGCT

GAGTCC- $3^{\prime}$

$M l x: 5^{\prime}$-GAGGACAGTGATTATCAGCAGGA- ${ }^{\prime}$ and $5^{\prime}$-CATCCCTTCTCTTC TGTTCAGC-3

MondoA: $5^{\prime}$-GACTCGGACACAGACGAGC- $3^{\prime}$ and $5^{\prime}$-GGAGAGGACACC ATGAAGTGG-3'

Immunostaining. For immunocytochemistry, cells were plated on $0.1 \%$ gelatin-coated Cell Disks (MS-92132, SUMITOMO BAKELITE Co., Tokyo, Japan) and cultured for an appropriate period. Then the cells were fixed with $4 \%$ paraformaldehyde for $20 \mathrm{~min}$ at room temperature. After extensive washes with phosphate-buffered saline (PBS), the cells were permeabilized and blocked in blocking buffer (PBS containing $0.3 \%$ Triton X-100, and $5 \%$ fetal bovine serum) for $60 \mathrm{~min}$ at room temperature. Subsequently, the cells were washed with PBS and then incubated with primary antibodies in blocking buffer at $4{ }^{\circ} \mathrm{C}$ overnight. After three washes with PBS, the cells were incubated with the appropriate Alexa Fluor dye-conjugated secondary antibodies (Invitrogen) and $3 \mu \mathrm{g} \mathrm{ml}^{-1}$ $4^{\prime}, 6$-diamidino-2-phenylindole (DAPI) in blocking buffer at $37^{\circ} \mathrm{C}$ for $1 \mathrm{~h}$. The cells were washed and observed under a confocal laser scanning microscope (TCS SP8, Leica Microsystems) and analysed with Image J computer software. For immunohistochemistry, male testes and the genital ridges of male and female siblings were isolated from 8 -week-old male mice and $13.5 \mathrm{dpc}$ embryos, respectively. After fixing with $4 \%$ paraformaldehyde, the tissues were embedded in optimal cutting temperature (OCT) compound and frozen. Sections prepared from the frozen tissues were mounted on glass slides and subjected to immunostaining procedures as described above. The primary antibodies used were as follows: anti-SYCP1 (1:400, ab15090), anti-SYCP3 (1:400, ab97672), anti-STRA8 (1:400, ab49602), anti-REC8 (1:100, ab192241) and anti-Aurora B (1:1,000, ab2254) from Abcam; anti-MAX (1:100, sc-197), anti-L3mbtl (1:100, sc-134879) and anti-PLZF (1:100, sc28319) from Santa Cruz Biotechnology; 5-methylcytosine $(1: 100,61255)$ and 5-hydroxymethylcytosine (1:100, 39791) from Active Motif; anti- $\gamma \mathrm{H} 2 \mathrm{AX}(1: 1,000$, \#2577, Cell Signaling Technology); anti-cKIT $(1: 100,553352)$ from BD Pharmingen; and Anti-Aurora A/B/C (1:300, BS-2445R) from Bioss Antibodies.

Estimation of meiotic stages from SYCP3-staininig length. Germ cells in preleptotene, leptotene, zygotene, pachytene and diplotene stages were identified the ordinary way, with testis sections immunostained and counterstained with antiSYCP3 antibody and DAPI, respectively. Subsequently, actual lengths of SYCP3staininig regions were measured with respect to five representative chromosomes of each cell. Some cells with large dots, the width of which was more than $1.5 \mu \mathrm{m}$, often found in cells at preleptotene and leptotene stages, were eliminated from the analyses. Exploration of the relationship between averages of these measurements and meiotic stages led to the following criteria: preleptotene-like, $<1.2 \mu \mathrm{m}$; leptotene-like, $1.2 \sim 3.0 \mu \mathrm{m}$; zygotene-like, $3.0 \sim 7.5 \mu \mathrm{m}$; and pachytene or diplotenelike, $>7.5 \mu \mathrm{m}$. This method did not allow us to distinguish between pachytene and diplotene stages. These criteria were used to assign the stages of meiosis-like cells from Max-null ESCs. For details, see Supplementary Fig. 3.

Gene disruption. The CRISPR/Cas9 system was used to conduct homozygous knockout of Stra8 and Blimp1 genes. The following oligonucleotides were used to edit exon 2 and exon 3 of Stra 8 and Blimp1 genes, respectively.

Stra8: 5'-CACCGATAATGGCCACCCCTGGAGA- ${ }^{\prime}$ and $5^{\prime}$-AAACTCTC CAGGGGTGGCCATTATC- $3^{\prime}$

Blimp 1: 5'-CACCGATCATGAAAATGGACATGG-3' ${ }^{\prime}$ and $5^{\prime}$-AAACCCATGT CCATTTTCATGAT $\overline{\mathrm{C}-3^{\prime}}$

Sequences from the original genomic DNA are underlined. The oligonucleotides were annealed and subcloned into the Bbs1 site of the pX330U6-Chimeric_BB_CBh-hSpCas9 vector ${ }^{44}$. The vector was then co-transfected with pCAG-IP into Max-null ESCs using lipofectamine 2000 (Invitrogen, Life Technology) and then subjected to puromycin selection. The resulting ESC 
colonies were individually cloned and used to prepare cell stocks and genomic DNA. Subsequently, PCRs were conducted to identify clones with homozygous knockout by direct DNA sequencing of the PCR products. Details of mutations in Stra 8 and Blimp1 genes are shown in Fig. 4a and Supplementary Fig. 8, respectively.

Lentivirus-mediated knockdown. Lentivirus-mediated knockdown of the expression of $M y c$ superfamily genes and the $L 3 m b t l 2$ gene was conducted as described previously ${ }^{45}$. Sequences used for knockdown are shown in Supplementary Table 1 .

ChIP-PCR analyses. ChIP analyses were done with Dox-untreated and -treated Max-null ESCs for 4 days using antibodies against MAX or L3MBTL2. Primers used for amplifying promoter regions of Ddx4, Slc25a31 and Sycp3 genes, which were described by Maeda et al. ${ }^{11}$, Okashita et al. ${ }^{46}$ and Yamaguchi et al. ${ }^{28}$, respectively, are as follows:

Ddx4: $5^{\prime}$-ACTCACCTCTCCGCTCCAG- $3^{\prime}$ and $5^{\prime}$-GTCGCCTGATGCTAT TTGTTGT- $3^{\prime}$

Slc25a31: $5^{\prime}$-AAAGCTGCTGTGCACTGATTG- $3^{\prime}$ and $5^{\prime}$-CTGAGGATGCTG GGAGAACAG-3'.

Sycp3: $5^{\prime}$-GGGGCCGGACTGTATTTACT-3' and $5^{\prime}$-CTCCCCCATCTCCTT ACCTC- $3^{\prime}$.

DNA microarray and GO analyses. Biotin-labelled cRNA was synthesized and then hybridized to Affymetrix GeneChip Mouse Genome 4302.0 arrays (Affymetrix) according to the manufacturer's instructions. Microarray expression data were background subtracted and normalized by the robust multi-array analysis method using statistical software R. GO analyses were conducted using DAVID web tools (http://david.abcc.ncifcrf.gov). To eliminate redundancy, the selected GO terms were subjected to further analyses using AmiGO1 (http://amigol.geneontology.org/cgi-bin/amigo/go.cgi) and REVIGO (http://revigo.irb.hr) web sites.

DNA blot and bisulfite sequencing analyses. DNA blot analyses of global changes in $5 \mathrm{mC}$ and $5 \mathrm{hmC}$ due to Max expression ablation in ESCs were performed using genomic DNAs from Dox-untreated and -treated Max-null ESCs for 8 days as described by Blaschke et al. ${ }^{29}$. Bisulfite sequencing analyses of Snrpn, Igf $2 r$ and $\mathrm{H} 19$ genes were performed with the same set of genomic DNAs using the following oligonucleotides, which are described by Lucifero et al. ${ }^{47}$

Snrpn first PCR: $5^{\prime}$-TATGTAATATGATATAGTTTAGAAATTAG- ${ }^{\prime}$ and $5^{\prime}$-A ATAAACCCAAATCTAAAATATTTTAATC- $3^{\prime}$

Snrpn second PCR: $5^{\prime}$-AATTTGTGTGATGTTTGTAATTATTTGG- ${ }^{\prime}$ and

5'-ATAAAATACACTTTCACTACTAAAATCC- $3^{\prime}$

Igf2r first PCR: $5^{\prime}$-TTAGTGGGGTATAAAAGGTTAATGAG-3 ${ }^{\prime}$ and $5^{\prime}$-AAAT

ATCCTAAAAATACAAACTACAC- $3^{\prime}$

Igf $2 r$ second PCR: $5^{\prime}$-GTGTGGTATTTTTATGTATAGTTAGG-3' and $5^{\prime}$-AA

ATATCCTAAAAATACAAACTACAC- $3^{\prime}$

H19 first PCR: ${ }^{\prime}$-GAGTATTTAGGAGGTATAAGAATT- $3^{\prime}$ and ${ }^{\prime}$-ATCAAA

AACTAACATAAACCCCT-3'

H19 second PCR: 5'-GTAAGGAGATTATGTTTATTTTTGG- $3^{\prime}$ and $5^{\prime}$-CCTC

ATTAATCCCATAACTAT- $3^{\prime}$

Uncropped versions of western blots shown in Fig. $4 \mathrm{c}$ and Supplementary

Fig. 5a are shown in Supplementary Fig. 14a,b, respectively.

\section{References}

1. Ginsburg, M., Snow, M. H. \& McLaren, A. Primordial germ cells in the mouse embryo during gastrulation. Development 110, 521-528 (1990).

2. Brar, G. A. \& Amon, A. Emerging roles for centromeres in meiosis I chromosome segregation. Nat. Rev. Genet. 9, 899-910 (2008).

3. Menke, D. B., Koubova, J. \& Page, D. C. Sexual differentiation of germ cells in XX mouse gonads occurs in an anterior-to-posterior wave. Dev. Biol. 262, 303-312 (2003).

4. Baltus, A. E. et al. In germ cells of mouse embryonic ovaries, the decision to enter meiosis precedes premeiotic DNA replication. Nat. Genet. 38, 1430-1434 (2006).

5. Anderson, E. L. et al. Stra8 and its inducer, retinoic acid, regulate meiotic initiation in both spermatogenesis and oogenesis in mice. Proc. Natl Acad. Sci. USA 105, 14976-14980 (2008).

6. Koubova, J. et al. Retinoic acid activates two pathways required for meiosis in mice. PLoS Genet. 10, e1004541 (2014).

7. Koubova, J. et al. Retinoic acid regulates sex-specific timing of meiotic initiation in mice. Proc. Natl Acad. Sci. USA 103, 2474-2479 (2006).

8. Bowles, J. et al. Retinoid signaling determines germ cell fate in mice. Science 312, 596-600 (2006).

9. Ohta, K., Lin, Y., Hogg, N., Yamamoto, M. \& Yamazaki, Y. Direct effects of retinoic acid on entry of fetal male germ cells into meiosis in mice. Biol. Reprod. 83, 1056-1063 (2010).
10. Raverdeau, M. et al. Retinoic acid induces Sertoli cell paracrine signals for spermatogonia differentiation but cell autonomously drives spermatocyte meiosis. Proc. Natl Acad. Sci. USA 109, 16582-16587 (2012).

11. Maeda, I. et al. Max is a repressor of germ cell-related gene expression in mouse embryonic stem cells. Nat. Commun. 4, 1754 (2013).

12. Kanatsu-Shinohara, M. et al. Long-term proliferation in culture and germline transmission of mouse male germline stem cells. Biol. Reprod. 69, 612-616 (2003).

13. Kanatsu-Shinohara, M. et al. Genetic and epigenetic properties of mouse male germline stem cells during long-term culture. Development 132, 4155-4163 (2005).

14. Gao, Z. et al. PCGF homologs, CBX proteins, and RYBP define functionally distinct PRC1 family complexes. Mol. Cell 45, 344-356 (2012).

15. Qin, J. et al. The polycomb group protein L3mbtl2 assembles an atypical PRC1-family complex that is essential in pluripotent stem cells and early development. Cell Stem Cell 11, 319-332 (2012).

16. Hishida, T. et al. Indefinite self-renewal of ESCs through Myc/Max transcriptional complex-independent mechanisms. Cell Stem Cell 9, 37-49 (2011).

17. Zalzman, M. et al. Zscan4 regulates telomere elongation and genomic stability in ES cells. Nature 464, 858-863 (2010).

18. Macfarlan, T. S. et al. Embryonic stem cell potency fluctuates with endogenous retrovirus activity. Nature 487, 57-63 (2012).

19. Heyting, C. Synaptonemal complexes: structure and function. Curr. Opin. Cell Biol. 8, 389-396 (1996).

20. Kee, K., Angeles, V. T., Flores, M., Nguyen, H. N. \& Reijo Pera, R. A. Human DAZL, DAZ and BOULE genes modulate primordial germ-cell and haploid gamete formation. Nature 462, 222-225 (2009).

21. Meuwissen, R. L. et al. A coiled-coil related protein specific for synapsed regions of meiotic prophase chromosomes. EMBO J. 11, 5091-5100 (1992).

22. Ollinger, R., Alsheimer, M. \& Benavente, R. Mammalian protein SCP1 forms synaptonemal complex-like structures in the absence of meiotic chromosomes. Mol. Biol. Cell 16, 212-217 (2005).

23. de Vries, F. A. et al. Mouse Sycp1 functions in synaptonemal complex assembly, meiotic recombination, and XY body formation. Genes Dev. 19, 1376-1389 (2005).

24. Tang, C. J., Lin, C. Y. \& Tang, T. K. Dynamic localization and functional implications of Aurora-C kinase during male mouse meiosis. Dev. Biol. 290, 398-410 (2006).

25. Seisenberger, S. et al. The dynamics of genome-wide DNA methylation reprogramming in mouse primordial germ cells. Mol. Cell 48, 849-862 (2012).

26. Ficz, G. et al. FGF signaling inhibition in ESCs drives rapid genome-wide demethylation to the epigenetic ground state of pluripotency. Cell Stem Cell 13, 351-359 (2013).

27. Leung, D. et al. Regulation of DNA methylation turnover at LTR retrotransposons and imprinted loci by the histone methyltransferase Setdb1. Proc. Natl Acad. Sci. USA 111, 6690-6695 (2014).

28. Yamaguchi, S. et al. Tet1 controls meiosis by regulating meiotic gene expression. Nature 492, 443-447 (2012).

29. Blaschke, K. et al. Vitamin C induces Tet-dependent DNA demethylation and a blastocyst-like state in ES cells. Nature 500, 222-226 (2013).

30. Hsu, P. D., Lander, E. S. \& Zhang, F. Development and applications of CRISPR-Cas9 for genome engineering. Cell 157, 1262-1278 (2014).

31. Ying, Q. L. et al. The ground state of embryonic stem cell self-renewal. Nature 453, 519-523 (2008).

32. Hackett, J. A. \& Surani, M. A. Regulatory principles of pluripotency: from the ground state up. Cell Stem Cell 15, 416-430 (2014).

33. Magnusdottir, E. \& Surani, M. A. How to make a primordial germ cell. Development 141, 245-252 (2014).

34. Ohinata, Y. et al. Blimp1 is a critical determinant of the germ cell lineage in mice. Nature 436, 207-213 (2005).

35. Nakaki, F. et al. Induction of mouse germ-cell fate by transcription factors in vitro. Nature 501, 222-226 (2013).

36. Amati, B. et al. Oncogenic activity of the c-Myc protein requires dimerization with Max. Cell 72, 233-245 (1993).

37. Kanatsu-Shinohara, M. \& Shinohara, T. Spermatogonial stem cell self-renewal and development. Annu. Rev. Cell Dev. Biol. 29, 163-187 (2013).

38. Baudino, T. A. \& Cleveland, J. L. The Max network gone mad. Mol. Cell. Biol. 21, 691-702 (2001)

39. Feng, C. W., Bowles, J. \& Koopman, P. Control of mammalian germ cell entry into meiosis. Mol. Cell. Endocrinol. 382, 488-497 (2014).

40. Kagiwada, S., Kurimoto, K., Hirota, T., Yamaji, M. \& Saitou, M. Replicationcoupled passive DNA demethylation for the erasure of genome imprints in mice. ЕMBO J. 32, 340-353 (2013).

41. Kim, J. et al. A Myc network accounts for similarities between embryonic stem and cancer cell transcription programs. Cell 143, 313-324 (2010). 
42. Kanatsu-Shinohara, M., Onoyama, I., Nakayama, K. I. \& Shinohara, T. Skp1-Cullin-F-box (SCF)-type ubiquitin ligase FBXW7 negatively regulates spermatogonial stem cell self-renewal. Proc. Natl Acad. Sci. USA 111, 8826-8831 (2014)

43. Yokobayashi, S. et al. PRC1 coordinates timing of sexual differentiation of female primordial germ cells. Nature 495, 236-240 (2013).

44. Cong, L. et al. Multiplex genome engineering using CRISPR/Cas systems. Science 339, 819-823 (2013).

45. Hishida, T. et al. Sirt1, p53, and p38(MAPK) are crucial regulators of detrimental phenotypes of embryonic stem cells with Max expression ablation. Stem Cells 30, 1634-1644 (2012).

46. Okashita, N. et al. PRDM14 promotes active DNA demethylation through the ten-eleven translocation (TET)-mediated base excision repair pathway in embryonic stem cells. Development 141, 269-280 (2014).

47. Lucifero, D., Mertineit, C., Clarke, H. J., Bestor, T. H. \& Trasler, J. M. Methylation dynamics of imprinted genes in mouse germ cells. Genomics 79, 530-538 (2002).

\section{Acknowledgements}

We thank Dr Minoru S.H. Ko and Tomoko Okuda for the reporter plasmid bearing the Zscan4 gene promoter and technical assistance, respectively. We are also indebted to Drs Takashi Shinohara, Mitinori Saitou, Hiroyuki Sasaki and Hiroaki Ohishi for their helpful discussions. This study was supported in part by the Ministry of Education, Culture, Sports, Science and Technology (MEXT) of Japan, and primarily by a Grant-in-Aid for the Support Project of Strategic Research at Private Universities to the Saitama Medical University, Research Center for Genomic Medicine. A.O. is the recipient of grants from the Japan Society for the Promotion of Science (JSPS) KAKENHI (grants numbers 25293082, 25670147 and 26112512). A.S. is supported by a grant from JSPS KAKENHI (grant number 26670162). Work in the laboratory of J.C.I.B. was supported by the G. Harold and Leila Y. Mathers Charitable Foundation, Universidad Católica San Antonio de Murcia (UCAM), and The Leona M. and Harry B. Helmsley Charitable Trust (2012-PG-MED002).

\section{Author contributions}

A.S.: conception and design, collection and assembly of data, data analysis and interpretation and manuscript writing; M.H., T.H., D.O., A.U., M.N., Y.N. and Y.M. (Yosuke Mizuno): collection and assembly of data, and data analysis and interpretation; Y.O.: data interpretation, and financial and administrative support; Y.M. (Yasuhisa Matsui): conception and design, and data interpretation; J.W.: conception and design, data interpretation and manuscript writing; J.C.I.B. and A.O.: conception and design, interpretation, manuscript writing, financial and administrative support and final approval of manuscript.

\section{Additional information}

Accession code: DNA microarray data used in Fig. 5a have been deposited in the NCBI Gene Expression Omnibus under accession number GSE65700.

Supplementary Information accompanies this paper at http://www.nature.com/ naturecommunications

Competing financial interests: The authors declare no competing financial interests.

Reprints and permission information is available online at http://npg.nature.com/ reprintsandpermissions/

How to cite this article: Suzuki, A. et al. Loss of MAX results in meiotic entry in mouse embryonic and germline stem cells. Nat. Commun. 7:11056 doi: 10.1038/ncomms11056 (2016).

(c) (i) This work is licensed under a Creative Commons Attribution 4.0 International License. The images or other third party material in this article are included in the article's Creative Commons license, unless indicated otherwise in the credit line; if the material is not included under the Creative Commons license, users will need to obtain permission from the license holder to reproduce the material. To view a copy of this license, visit http://creativecommons.org/licenses/by/4.0/ 